Revue d'histoire de l'Amérique française REVUE D'HISTOIRE
DE L'AMÉRIQUE FRANCCAISE

\title{
Index par auteur de compte rendu, de note critique, de débat ou de note bibliographique
}

Index, 1997

Index des volumes 41 à 50 (été 1987 - printemps 1997)

URI : https://id.erudit.org/iderudit/305566ar

DOI : https://doi.org/10.7202/305566ar

Aller au sommaire du numéro

Éditeur(s)

Institut d'histoire de l'Amérique française

\section{ISSN}

0035-2357 (imprimé)

1492-1383 (numérique)

Découvrir la revue

Citer ce document

(1997). Index par auteur de compte rendu, de note critique, de débat ou de note bibliographique. Revue d'histoire de l'Amérique française, 129-160.

https://doi.org/10.7202/305566ar d'utilisation que vous pouvez consulter en ligne. 
(DEB) indique un débat

(NB) indique une note bibliographique.

(NC) indique une note critique.

A

ADAMCZYK, Georges, Annick GERMAIN et Jean-Claude MARSAN, dir., Aménager l'urbain, de Montréal à San Francisco. Politiques et design urbains, 42, 2: 285-287

ALBERT, Mathieu, Iro TEMBECK, Danser à Montréal. Germination d'une histoire chorégraphique, 46, 3: 543546

ALLAIRE, Gratien, David V. BURLEY, J. Scott HAMILTON et Knut R. FLADMARK, Prophecy of the Swan: the Upper Peace River Fur Trade of 1794-1823, 50, 3: 438-440; Marcel DURIEUX, Un héros malgré lui, 41, 1: 97-98; Raymond HUEL, dir.; Guy LACOMBE, coll., Études oblates de l'Ouest 2, 47, 4: 561-564; Paul C. THISTLE, Indian-European Trade Relations in the Lower Saskatchewan River Region to 1840, 42, 1: 119-121

ANCTIL, Pierre, Irving ABELLA, $A$ Coat of Many Colours. Two Centuries of Jewish Life in Canada, 44, 3: 417-420

ANTOLIN, Francisco, John A. DICKINSON et Marianne MAHN-LOT, 14921992: les Européens découvrent l'Amérique, 46, 4: 680-681

ARMSTRONG, Christopher, Claude BELLAVANCE, Shawinigan Water and Power, 1898-1963. Formation et déclin d'un groupe industriel au Québec, 49, 1: 87-89; Garth STEVENSON, Ex Uno Plures: Federal-Provincial Relations in Canada, 1867-1896, 49, 1: 108-110
B

BAILLARGEON, Denyse, Audrey KOBAYASHI, dir., Women, Work and Place, 50, 2: 281-283; Marie-Paule MALOUIN, dir., L'univers des enfants en difficulté au Québec entre 1940 et 1960, 50, 3: 466-469

BALIKCI, Asen, Gérard DUHAIME, De l'igloo au HLM. Les Inuit sédentaires et l'Etat-providence (NB), 41, 4: 633-634

BARBER, Marilyn, Dionne BRAND, No Burden to Carry: Narratives of Black Working Women in Ontario 1920s to 1950s, 47, 1: 101-103

BARRIĖRE, Mireille, Jean-Paul BAILLARGEON, dir., Les pratiques culturelles des Québécois: une autre image de nous-mêmes, 41, 1: 104-106

BASKERVILLE, Peter, Michael J. PIVA, The Borrowing Process: Public Finance in the Province of Canada, 1840-1867, 47, 2: 294-295

BEAUDOIN, André, Allan MOSCOVITCH et Jim ALBERT, dir., The Benevolent State. The Growth of Welfare in Canada, 42, 3: 477-479

BEAUDOIN, Réjean, Gérard BOUCHARD et Yvan LAMONDE, dir., Québécois et Américains. La culture québécoise aux XIX et $X X^{e}$ siècles, 50, 1: 105-106

BEAUDREAU, Sylvie, Félix ALBERT, Immigrant Odyssey: a French Canadian Habitant in New England, 45, 3: 431-433

BEAUDRY, Claude, Élisabeth GALLATMORIN, Jean Girard, musicien en Nouvelle-France: Bourges, 1696 Montréal, 1765 (NB), 48, 4: 591-592

BEAULIEU, Alain, Lucien CAMPEAU, s.j., La mission des jésuites chez les $\mathrm{Hu}$ rons, 1634-1650, 41, 2: 249-253; Élisabeth TOOKER, Ethnographie des Hurons, 1615-1649, 43, 2: 279-281 
BEAUREGARD, France, Robert TOUPIN, s.j., Arpents de neige et Robes noires. Brève relation sur le passage des jésuites en Nouvelle-France aux $X V I T^{e}$ et XVIIT siècles, 46, 2: 354-356

BEHIELS, Michael D., Serge DENIS, Le long malentendu. Le Québec vu par les intellectuels progressistes au Canada anglais, 1970-1991, 47, 3: 421-424

BÉLANGER, Louis, Mariel O'NEILL$\mathrm{KARCH}$, Théâtre franco-ontarien. Espaces ludiques, 48, 1: 111-112

BÉLANGER, Réal, FAUCHER DE SAINT-MAURICE, La question $d u$ jour. Resterons-nous Français? (NB), 45, 4: 631-632; Jean PROVENCHER, Chronologie du Québec (NB), 47, 2: 303-305; P. B. WAITE, The Man from Halifax. Sir John Thompson Prime Minister, 41, 3: 432-433

Bellavance, Claude, Philippe FAUCHER et Johanne BERGERON, $\mathrm{Hy}$ dro-Québec, la société de l'heure de pointe, 41, 2: 283-286; Keith R. FLEMING, Power at Cost. Ontario Hydro and Rural Electrification, 1911-1958, 47, 2: 273-276; Michèle MARTIN, "Hello, Central?» Gender, Technology, and Culture in the Formation of Telephone Systems, 46, 3: 539-541

BELLEFLEUR, Michel, Gilles BOURQUE et Jules DUCHASTEL, Restons traditionnels et progressifs. Pour une nouvelle analyse du discours politique: le cas du régime Duplessis au Québec, 42, 3: 447-449; Marcel FOURNIER, L'entrée dans la modernité: science, culture et société au Québec, 41, 3: 438-440

BENOIT, Jean, George BERVIN, Québec au XIX siècle. L'activité économique des grands marchands, 46, 2: 293 295

BENOIT, Monique, Paolo CARILE, Lo sguardo impedito - Studi sulle relazioni di viaggio in "Nouvelle-France» e sulla letteratura popolare, 42, 2: 268-270
BERNARD, André, Claude MORIN, L'art de l'impossible. La diplomatie québécoise depuis 1960, 41, 4: 626-627

BERNIARD, Jean-Paul, Allan GREER et Ian RADFORTHS, dir., Colonial Leviathan: State Formation in MidNineteenth Century Canada, 47, 3: 439-442; Allan GREER, The Patriots and the People: the Rebellion of 1837 in Rural Lower Canada, 49, 3: 430433; Jean HAMELIN et al., Guide du chercheur en histoire canadienne, 41, 2: 245-246; Jean LAMARRE, Le devenir de la nation québécoise selon Maurice Séguin, Guy Frégault et Michel Brunet (1944-1969), 48, 3: 443-446

BERNARD, Roger, Dean LOUDER, dir., Le Québec et les francophones de la Nouvelle-Angleterre, 47, 2: 288-291

BERNIER, Jacques, Jay CASSEL, The Secret Plague: Venereal Disease in Canada 1838-1939, 42, 3: 452-454; Jean-Pierre GOUBERT et Othmar KEEL, dir., Polysémie de la santé: institutions et pratiques sociales en France et au Québec (1750-1980), 50, 1: $120-121$

BERNIER, Serge, T. Robert FOWLER, Valour on Juno Beach. D-Day - June 6, 1944, 48, 4: 554-555; Paul LÉTOURNEAU, Le Canada et l'OTAN après 40 ans, 1949-1989, 47, 1: 122-124

BERTHIAUME, Pierre, Joseph L. PEYSER., dir., Letters from New France: the Upper Country, 1686-1783, 46, 4: 711.712

BIBEAU, Pierre, Robert McGHEE, Le Canada au temps des aventuriers, 47, 1: $130-131$

BISCHOFF, Peter, René HARDY et Guy TRÉPANIER, Bibliographie de la Mauricie (NB), 46, 2: 369-370

BLAY, Jacqueline, Richard LAPOINTE et Lucille TESSIER, Histoire des Franco-Canadiens de la Saskatchewan, 41, 1: 89-91; Estelle MITCHELL, Les Souurs Grises de Montréal à la RivièreRouge, 1844-1984 (NB), 42, 1: 125 127 
BOGLIONI, Pierre, Brigitte CAULIER, L'eau et le sacré. Les cultes thérapeutiques autour des fontaines en France, du Moyen Age à nos jours, 45, 4: 604606

BOIS, Hélène, Simon LAPOINTE, L'influence de la gauche catholique française sur l'idéologie de la CTCC-CSN de 1948 à 1964, 50, 4: 627-629

BOIVIN, Aurélien, Jacques CASTONGUAY, Philippe Aubert de Gaspé, seigneur et homme de lettres, 45, 4: 601603

BOSHER, J. F., Louise DECHÊNE, Le partage des subsistances au Canada sous le Régime français, 49, 1: 95-97

BOUCHARD, Gérard, $\dot{A}$ propos de Quelques arpents d'Amérique de Gérard Bouchard (DEB), Christian DESSUREAULT et Thomas WIEN, 50, 3: 401-435

BOUCHER, Romuald, Gérald C. BOUDREAU, Le père Sigogne et les Acadiens du sud-ouest de la NouvelleÉcosse, 46, 4: 667-669

BOUDREAU, Claude, Ruth McKENZIE, The St. Lawrence Survey Journals of Captain Henry Wolsey Bayfield, 1829 1853, 42, 1: 110-113

BOUDREAULT, Sylvain, Les Cahiers des Dix, $n^{\circ} 46,46,2:$ 298-300

BOURASSA, Claire, Louise NORMANDEAU et Victor PICHÉ, Les populations amérindiennes et inuit du Canada. Aperçu démographique (NB), 41, 1: $109-10$

BOURASSA-TRÉPANIER, Juliette, Marie-Thérèse LEFEBVRE, La création musicale des femmes au Québec, 45, 4: 609-611

BOUVIER, Félix, Robert COMEAU et Lucille BEAUDRY, dir., André Laurendeau, un intellectuel d'ici, 45, 2: 272-274

BRADBURY, Bettina, Marjorie Griffin COHEN, Women's Work, Markets, and Economic Development in NineteenthCentury Ontario, 42, 4: 607-609; Christopher ARMSTRONG et H. V. NELLES, Monopoly's Moment: The
Organization and Regulation of Canadians Utilities, 1830-1930, «L'heur des monopoles» (NC), 41, 3: 403-408

BRAEN, André, Serge BERNIER et Jean PARISEAU, Les Canadiens français et le bilinguisme dans les Forces armées canadiennes, 2: 1969-1987: langues officielles: la volonté gouvernementale et la réponse de la Défense nationale, 47, 1: 96-98

BRAULT, Marie-Marthe T., Réal BRISSON, La mort au Québec. Dossier exploratoire, 43, 3: 405-407

BRISEBOIS, Michel, Jacques MICHON, dir., Édition et pouvoirs, 50, 2: 299-300

BRODEUR, Jean-Paul, Danielle LACASSE, La prostitution féminine à Montréal: 1945-1970, 50, 1: 122-125

BRODEUR, Raymond, Nadine-Josette CHALINE, René HARDY et Jean ROY, prés., La Normandie et le Québec vus du presbytère, 42, 3: 455-456; Donat LEVASSEUR, o.m.i., Les Oblats de Marie Immaculée dans l'Ouest et le Nord du Canada, 18451967, 50, 2: 292-294; Guy-Marie OURY, Notre héritage chrétien. Histoire religieuse populaire du Canada, 45, 3: 455-458

BROWN, Wallace, Neil MacKINNON, This Unfriendly Soil: the Loyalist Experience in Nova Scotia 1783-1791, 41, 3: $423-426$

BRUN, Josette, Sally ROSS et J.-Alphonse DEVEAU, Les Acadiens de la Nouvelle-Écosse hier et aujourd'hui, 50, 4: 629-630

BRUNET, Manon, Jeanne-d'Arc LORTIE, Pierre SAVARD et Paul WYCZYNSKI, Les textes poétiques du Canada français, 1606-1867, 1: 16061806, 42, 1: 107-110

BUCKNER, Phillip A., Janet AJZENSTAT, The Political Thought of Lord Durham, 43, 1: 93-95

BURGESS, Joanne, Bettina BRADBURY, Familles ouvrières à Montréal. Age, genre et survie quotidienne pendant la phase d'industrialisation, 49, 1: 
91-95; Craig HERON et Robert STOREY, dir., On the Job. Confronting the Labour Process in Canada, 41, 4: 600603; Claudette LACELLE, Les domestiques en milieu urbain canadien au XIX siècle, 43, 1: 115-116

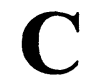

CADOTTE, Marcel, Michèle BENOIT et Roger GRATTON, Pignon sur rue. Les quartiers de Montréal (NB), 46, 3: 549550; Josette MICHAUD, Les auvres $d u$ temps. Le Vieux-Montréal, 46, 4: 710

CADRIN, Paul, J. Paul GREEN et Nancy F. VOGAN, Music Education in Canada: a Historical Account, 46, 2: $317-$ 320

CAMPEAU, Lucien, Monumenta Novee Francice, 4: Les grandes épreuves (1638-1640) (NC), 44, 1: 97-103; Léandre POIRIER, Les Franciscains Récollets en Nouvelle-France, 16151848 (NB), 41, 3: 457

CANUEL, Alain, Susan SHEETSPYENSON, Cathedrals of Science: the Development of Colonial Natural History Museums During the Late Nineteenth Century, 42, 4: 636-639

CARDIN, Jean-François, Donald AVERY et Roger HALL, dir., Coming of Age. Readings in Canadian History Since World War II, 50, 2: 243-246; CONSEIL DES TRAVAILLEUSES ET TRAVAILLEURS DU MONTRÉAL MÉTROPOLITAIN, Cent ans de solidarité. Histoire du CTM 18861986, 41, 4: 616-617; John A. DICKINSON et Brian YOUNG, Brève histoire socio-économique du Québec, 47, 1: 110-112; John SAYWELL, Canada: Pathways to the Present, 49, 2: 289291; Cyril W. STRONG, My Life as a Newfoundland Union Organizer: the
Memoirs of Cyril W. Strong, 19121987, 42, 2: 308-310; Merrily WEIS$\mathrm{BORD}$, Le rêve d'une génération. Les communistes canadiens, les procès d'espionnage et la guerre froide, 43, 3: 436-438

CARDIN, Martine, Montréal citée. 60 ans d'histoire à travers les archives sonores (1930-1990), 46, 4: 692-694

CARIE, Paul, Richard A. JARRELL, The Cold Light of Dawn: a History of Canadian Astronomy, 42, 3: 469-472

CARON, Daniel J., Denis VAUGEOIS, La fin des alliances franco-indiennes, enquête sur un sauf-conduit de 1760 devenu un traité en 1990, 50, 2: 315317

CARRIER, Jean-Pierre, Daniel JACQUES, Les humanités passagères: considérations philosophiques sur la culture politique québécoise, 46, 3: 535536

CASTONGUAY, René, Claude G. CHARRON, La partition du Québec: de lord Durham à Stéphane Dion, 50, 3: 443445; Robert COMEAU et Michel LÉVESQUE, Le Parti québécois: bibliographie rétrospective (NB), 45, 4: 630631; Robert COMEAU et Michel LÉVESQUE, Partis politiques et élections provinciales au Québec: bibliographie rétrospective (1867-1991) (NB), 47, 3: 460-461; Michel LÉVESQUE et Robert COMEAU, Le Parti libéral $d u$ Québec: bibliographie rétrospective (1867-1990) (NB), 45, 4: 630-631; Lawrence MARTIN, Chrétien, 1: the Will to Win, 50, 3: 470-471

CAUI_IER, Brigitte, Serge GAGNON, Mourir, hier et aujourd'hui. De la mort chrétienne dans la campagne québécoise au XIX siècle à la mort technicisée dans la cité sans Dieu, 41, 4: 599600

CAYA, Marcel, BIBLIOTHÈQUE DE L'ASSEMBLÉE NATIONALE, Dictionnaire des parlementaires du Québec, 1792-1992, 47, 3: 416-418; LouisAntoine DESSAULLES, édition criti- 
que par Yvan LAMONDE, 50, 1: 109110; Philippe FERLAND, Paul Gouin (NB), 47, 3: 461-463; Gilles GALLICHAN, texte établi par, Débats de l'Assemblée législative, $12^{e}$ Législature, $4^{e}$ session (NB), 50, 4: 635

CELLARD, André, Peter KEATING, $L a$ science du mal. L'institution de la psychiatrie au Québec, 1800-1914, 48, 1: 95-97

CHARBONNEAU, André, Gaston DESCHÊNES et Luc NOPPEN, L'Hôtel $d u$ Parlement témoin de notre histoire, 41 , 1: 83-85

CHARBONNEAU, Hubert, Roderic BEAUJOT, Population Change in Canada. The Challenges of Policy Adaptation, 46, 2: 292-293; Marcel FOURNIER, Les Français au Québec, 1765-1865. Un mouvement migratoire méconnu (NB), 49, 4: 600

CHARLAND, Jean-Pierre, L'histoire de l'éducation au Québec. Regard sur la production récente (NC), 50, 4: 599614; Jacques PORTES, Le Canada et le Québec au XX' siècle, 48, 4: 579-581; réplique, 49, 2: 297-298; réponse de Jean-Pierre CHARLAND, 49, 2: 298300 ; R. A. RICHARDSON et B. H. MacDONALD, Science and Technology in Canadian History: a Bibliography of Primary Sources, 42, 3: 482-483

CHARLES, Aline, Denis GOULET, Le commerce des maladies. La publicité des remèdes au début du siècle, 41, 3: 436-438

CHEVRIER, Daniel, José BENMOUYAL, Des Paléoindiens aux Iroquoiens en Gaspésie: six mille ans d'histoire, 42, 4: 600-602

CHOQUETTE, Robert, Roger GUINDON, Coexistence difficile. La dualité linguistique à l'Université d'Ottawa, 1: 1848-1898, 43, 4: 574-576; Simon LANGLOIS, dir., Identité et cultures nationales. L'Amérique française en mutation, 49, 4: 582-585; Jeanne d'Arc LORTIE, s.c.o., Lettres d'Élisabeth Bruyère, 1: 1839-1849, 43, 3: 415-416;
Jeanne d'Arc LORTIE, Lettres d'Élisabeth Bruyère, 2: 1850-1856, 46, 4: 709

CLERMONT, Norman, Hélène BÉDARD, Les Montagnais et la réserve de Betsiamites: 1850-1900, 42, 3: 443445; J. A. TUCK, La préhistoire des Provinces maritimes (NB), 41, 3: 456

CLICHE, Marie-Aimée, Pierre-Lionel LABERGE, Messire Gaspard Dufournel et l'histoire véritable de l'AngeGardien, de ses curés, de ses églises, de son trésor, 1664-1760, 47, 1: 118-120

COATES, Colin M., Mario BÉLAND, dir., La peinture au Québec 1820-1850: nouveaux regards, nouvelles perspectives, 47, 3: 411-414; Nicole GUILBAULT, Il était cent fois la Corriveau. Anthologie (NB), 50, 3: 485-486; L'EXPOSITION, La peinture au Québec 1820-1850: nouveaux regards, nouvelles perspectives, $47,3: 411-414$

CODIGNOLA, Luca, Lucien Campeau, dir., Monumenta Nova Francia, 4: Les grandes épreuves (1638-1640) (NC), 44, 1: 97-103

COLLARD, Chantal, Anne-Marie DESDOUITS, Le monde de l'enfance. Traditions du pays de Caux et du Québec, 44, 4: 595-596

COLLIN, Jean-Pierre, Gilles LAUZON, Habitat ouvrier et révolution industrielle: le cas du village Saint-Augustin, 43, 3: 412-415; Bryan P. MELNYK, Calgary Builds. The Emergence of an Urban Landscape, 1905-1914, 41, 1: 95-97

COLLIN, Johanne, Denis GOULET, Histoire de la Faculté de médecine de l'Université de Montréal, 1843-1993, 48, 1: 90-92

COLLINS, Anne Marie, COLLECTIF, Les chemins de la mémoire, 1: Monuments et sites historiques du Québec, 46, 2: 304-305

COMEAU, Michelle, Jean-Marc PIOTTE, La communauté perdue. Petite histoire des militantismes, 42, 1: 113-115

COMEAU, Paul-André, Vincent LEMIEUX, Le Parti libéral du Québec. 
Alliances, rivalités et neutralités, 48,1 : 104-106

CONRAD, Margaret, N. E. S. GRIFFITHS et G. A. RAWLYCK, dir., $M a-$ son Wade, Acadia and Quebec. The Perception of an Outsider, 47, 2: 278279; Naomi E. S. GRIFFITHS, The Contexts of Acadian History, 16861784, 47, 2: 278-279

COOK, Ramsay, David J. BERCUSON et Barry COOPER, Derailed: the Betrayal of the National Dream, 49, 1: 89-91; Martin L. FRIEDLAND, The Case of Valentine Shortis. A True Story of Crime and Politics in Canada, 41, 3: 434-435; Fernande ROY, Progrès, harmonie, liberté: le libéralisme des milieux d'affaires francophones à Montréal au tournant du siècle, 43, 1: 116119

COPP, Terry, Stephen J. HARRIS, $\mathrm{Ca}$ nadian Brass: the Making of a Professional Army (1860-1939), 43, 2: 265266

CORNETT, Norman F., Kenelm BURRIDGE, In the Way. A Study of Christian Missionary Endeavours, 46, 4: 670-671; Michael GAUVREAU, The Evangelical Century. College and Creed in English Canada from the Great Revival to the Great Depression, 46, 2: 309-311

CÔTÉ, André, ARCHIVES NATIONALES DU QUÉBEC, Archives d'origine privée conservées au Centre d'archives du Saguenay-Lac-Saint-Jean: guide (NB), 47, 3: 459-460

CÔTÉ, Luc, Pierre PAGÉ, Radiodiffusion et culture savante au Québec (19301960), 48, 2: 273-275

CÔTÉ, Sylvie, Huguette LAPOINTEROY, Charité bien ordonnée. Le premier réseau de lutte contre la pauvreté à Montréal au $19^{e}$ siècle, 41, 1: 85-87

COURVILLE, Serge, Louise DECHÊNE, Atlas historique du Canada, I: Des origines à 1800, "Mari Usque ad Mare - La grande saga canadienne» (NC), 42, 3: 429-439; Michael P. CONZEN,
Thomas A. RUMNEY et Graeme WYNN, A Scholar's Guide to Geographical Writing on the American and Canadian Past, 47, 1: 106-108

COUTURE, Claude, Pierre ANCTIL, Le Devoir, les Juifs et l'immigration, 43, 1: 96-98; Pierre ANCTIL, Le rendez-vous manqué. Les Juifs de Montréal face au Québec de l'entre-deux-guerres, 43, 1: 96-98; François CROUZET, L'économie britannique et le Blocus continental, 42, 4: 617-618; David ORCHARD, The Fight for Canada. Four Centuries of Resistance to American Expansionism, 48, 2: 271-273; Françoise TÉTU DE LABSADE, Le Québec, un pays, une culture, 44, 4: 613-615

COUTURE, Simon, Juliette BOURASSA-TRÉPANIER et Lucien POIRIER, Répertoire des données musicales de la presse québécoise, I: Canada, 1: 1764 1799, 45, 4: 596-598;

COUTURIER, Jacques Paul, L'Acadie des Maritimes (NC), 49, 2: 247-257; T. W. ACHESON, Saint John: the Making of a Colonial Urban Community, 42, 3: 441-443; Jean DAIGLE, L'Acadie des Maritimes: études thématiques des débuts à nos jours, 49, 2: 247-257

CRAIG, Béatrice, Margaret CONRAD, dir., Making Adjustments. Change and Continuity in Planter Nova Scotia, 175.9-1800, 46, 3: 512-513; Judith A. NORTON, comp., New England Planters in the Maritime Provinces of $\mathrm{Ca}$ nada, 1759-1800, 47, 4: 573-574

CROCHETIĖRE, Jacques, L'Église de Montréal, aperçus d'hier et d'aujourd'hui, 1836-1986, 41, 2: 269-271; LéoPaul HÉBERT, Le Québec de 1850 en lettres détachées (NB), 41, 3: 457-458

CROSS, Michael, Jane ERRINGTON, The Lion, the Eagle, and Upper Canada: a Developing colonial Ideology, 42, 4: 6:22-624

CUCCIOLETTA, Donald, André-G. BOURASSA et Jean-Marc LARRUE, Les nuits de la «Main»: cent ans de spectacles sur le boulevard Saint-Laurent 
(1891-1991), 47, 3: 415-416; Marcel JEAN, Le cinéma québécois, 46, 2: 324-326; Jean-Marc LARRUE, Le monument inattendu. Le MonumentNational 1893-1993, 48, 4: 573-574

CYR, Jean-Roch, Georges ARSENAULT, Les Acadiens de l'Île, 17201980, 43, 1: 99-101

D

D'ALLAIRE, Micheline, Nicole LAURIN, Danielle JUTEAU et Lorraine DUCHESNE, À la recherche d'un monde oublié. Les communautés religieuses de femmes au Québec de 1900 à 1970, 46, 2: 329-332; Nive VOISINE, Les Frères des Écoles chrétiennes au Canada, 2: Une ère de prospérité, 1880-1946, 47, 1: 141-143

DAGENAIS, Michèle, Jean-Pierre DUQUETTE, dir., Montréal, 1642-1992, 47, 3: 427-428; Michel LESSARD, dir., Montréal au $X X^{e}$ siècle. Regards de photographes, 49, 4: 585-587; Michel LESSARD, Montréal, métropole du Québec. Images oubliées de la vie quotidienne 1852-1910, 47, 4: 566568; Graham S. LOWE, Women in the Administrative Revolution. The Feminization of Clerical Work, 41, 4: 622624; Diane SAINT-PIERRE, L'évolution municipale du Québec des régions. Un bilan historique, 49, 2: 287-289

DAIGLE, Jean, C. J. TAYLOR, Negotiating the Past. The Making of Canada's National Historic Park and Sites, 45, 4: 624-626

DAIGLE, Johanne, Sylvie MURRAY, $\grave{A}$ la jonction du mouvement ouvrier et du mouvement des femmes: la Ligue auxiliaire de l'Association internationale des machinistes, Canada, 1903-1980, 44, 3: 439-441
DALLAIRE, Louis, Gérard BOUCHARD et Marc DeBRAEKELEER, Pourquoi des maladies héréditaires?, 47, 1: 100101

DANSEREAU, Bernard, Gregory S. KEALEY et Reg WHITAKER, dir., R.C.M.P. Security Bulletins. The Depression Years, Part II, 1935, 49, 2: 270-271; Gregory S. KEALEY et Reg WHITAKER, dir., R.C.M.P. Security Bulletins. The Early Years, 1919-1929, 48, 4: 568-569

DAVIDSON, Malcolm, J. Rick PONTING, Arduous Journey: Canadian Indians and Decolonizataion, 41, 2: 281283

DAWSON, Nelson-Martin, Louis-Antoine de BOUGAINVILLE; Roland LAMONTAGNE, dir., Écrits sur le Canada. Mémoires - Journal - Lettres, 48, 2: 245-247

De BONVILLE, Jean, Bernard DANSEREAU, L'avènement de la linotype: le cas de Montréal à la fin du XIX siècle, 46, 3: 516-517; Camil GIRARD, Un pays fragile: le Times de Londres et l'image du Canada (1908-1922), 48, 4: 559-561; Robert LAHAISE, dir., Le Devoir, reflet $d u 20^{\circ}$ siècle, 50, 1: 125127

De LAGRAVE, Jean-Paul, Roger LE MOINE, Deux loges montréalaises $d u$ Grand Orient de France (NB), 46, 2: 373-374

DECHÊNE, Louise, Gaston DESCHÊNES, L'année des Anglais. La Côte-duSud à l'heure de la Conquête (NB), 43, 2: 281-282

DELÂGE, Denys, Bruce G. TRIGGER, Les Indiens, la fourrure et les Blancs: Français et Amérindiens en Amérique $d u$ Nord, 45, 1: 126-128; Joseph ZITOMERSKY, French American-Native Americans in Eighteenth-Century French Colonial Louisiana. The Population Geography of the Illinois Indians, 1670s-1760s, 50, 3: 481-482 
DENIGER, Marc-André, Dennis GUEST, Histoire de la sécurité sociale au Canada, 49, 1: 101-103

DENIS, Serge, Charles TAYLOR, Rapprocher les solitudes. Écrits sur le fédéralisme et le nationalisme au Canada (NC), 47, 2: 257-263

DENNIE, Donald, Cornelius J. JAENEN, dir., Les Franco-Ontariens, 48, 4: 565568

DÉPATIE, Sylvie, John Irvine LITTLE, Crofters and Habitants. Settler Society, Economy and Culture in a Quebec Township, 1848-1881, 47, 1: 124-128

DESBIENS, Albert, Jean-François LISÉE, Dans l'ail de l'aigle: Washington face au Québec, 45, 1: 124-126; Yves ROBY, Les Franco-Américains de la Nouvelle-Angleterre, 1776-1930, 44, 4: 612-613

DÉSILETS, Andrée, Dictionnaire biographique du Canada, 13: 1901-1910 (NB), 49, 4: 599; Camil GIRARD, Question d'empire. Le Times de Londres et le Canada, 1908-1922, 43, 3: 410-411; Ged. MARTIN, dir., The Causes of Canadian Confederation (NB), 46, 3: 551-552; R. Gand MOYLES et Doug OWRAM, Imperial Dreams and Colonial Realities. British Views of Canada, 1880-1914, 43, 2: 270-271; Marcel TRUDEL, Mémoires d'un autre siècle, 41, 4: 629-631; Denis VAUGEOIS, Québec 1792. Les acteurs, les institutions et les frontières, 47, 2: 299-300

DESJARDINS, Rita, Aline CHARLES, Travail d'ombre et de lumière. Le bénévolat féminin à l'Hôpital Sainte-Justine, 1907-1960, 44, 4: 591-593; Nicolle FORGET, Francine HAREL-GIASSON et Francine SÉGUIN, Justine Lacoste-Beaubien et l'Hôpital SainteJustine, 50, 2: 268-269

DESLANDRES, Dominique, Luca CODIGNOLA, Guide des documents relatifs à l'Amérique du Nord française et anglaise dans les archives de la Sacrée Congrégation de la Propagande à
Rome, 1622-1799 (NB), 46, 2: 366367; Elizabeth RAPLEY, The Devotes. Women and Church in SeventeenthCentury France, 45, 2: 291-294

DESL.OGES, Yvon, Hélène BOURQUE, La maison de faubourg. L'architecture domestique des faubourgs Saint-Jean et Saint-Roch avant 1845, 46, 3: 506-508

DESROSIERS, Richard, Marcel PEPIN, Le nécessaire combat syndical, 42, 4: 635-636

DESSUREAULT, Christian, $\grave{A}$ propos de Quelques arpents d'Amérique de Gérard Bouchard (DEB), 50, 3: 401-435

DESSUREAULT, Christian, Gérald BEIRNIER et Daniel SALÉE, Entre l'ordre et la liberté. Colonialisme, pouvoir et transition vers le capitalisme dans le Québec du XIX siècle, 49, 4: 567-569; Gérard BOUCHARD, Quelques arpents d'Amérique. Population, économie, famille au Saguenay 18381971 (DEB), 50, 3: 401-435

DICKASON, Olive Patricia, Bruce G. TRIGGER, Toby MORANTZ et Louise DECHÊNE, dir., Le castor fait tout. Choix de textes présentés à la $5^{e}$ conférence nord-américaine sur la traite de la fourrure, 1985, 42, 3: 483486

DICKINSON, John A., Jacques CARTIE:R, Relations, 42, 1: 91-94; Claude CHAPDELAINE, La maison longue iroquoienne de Lanoraie (NB), 41, 2: 287-288; Luca CODIGNOLA, The Coldest Harbour of the Land. Simon Stock and Lord Baltimore's Colony in Newfoundland, 1621-1649, 43, 4: 569570; Roberta HAMILTON, Feudal Society and Colonization. The Historiography of New France, 42, 4: 629-632; William C. JAMES, A Fur Trader's Photographs. A. A. Chesterfield in the District of Ungava, 1901-4 (NB), 41, 4: 636; Edward S. ROGERS et Donald B. SMITH, dir., Aboriginal Ontario. Historical Perspectives on the First $\mathrm{Na}$ tions, 49, 2: 283-285; Roger SCHLESINGER et Arthur P. STABLER, dir., 
André Thevet's North America: a Sixteenth Century View, 42, 1: 91-94; Donald B. SMITH, From the Land of Shadows. The Making of Grey Owl, 44, 2: 283; André VACHON, Victorin CHABOT et André DESROSIERS, L'enracinement. Le Canada de $1700 \grave{a}$ 1760 (NB), 41, 4: 634-635; Robert S. WEDDLE, Mary Christine MORKOVSKY et Patricia GALLOWAY, dir., La Salle, the Mississippi, and the Gulf. Three Primary Documents, 42, 1: 91-94

DICKSON, Paul, David J. BERCUSON, Maple Leaf Against the Axis: Canada's Second World War, 50, 1: 103-104

DION, Stéphane, André BERNARD, Problèmes politiques. Canada et Québec, 48, 2: 241-242; François ROCHER, dir., Bilan québécois du fédéralisme canadien, 47, 1: 137-138

DIONNE, Bernard, Jean-François CARDIN et Jacques ROUILLARD, Guide des archives des Unions internationales à Montréal, 42, 3: 449-450; Bruno DESHAIES, Méthodologie de la recherche en sciences humaines, 47, 4: 552-555; Jacques ROUILLARD, Histoire du syndicalisme québécois, 43,3 : 425-428

DOLAN, Claire, Gérard BOUCHARD et al., dir., Famille, économie et société rurale en contexte d'urbanisation $\left(17^{e}-\right.$ $20^{\circ}$ siècle), «À propos de la société rurale en contexte d'urbanisation» (NC), 45, 1: 101-109; Joseph GOY, JeanPierre WALLOT et Rollande BONNAIN, Évolution et éclatement du monde rural. France-Québec, XVII$X X^{e}$ siècles, "Nouveaux besoins, nouvelles cibles? L'histoire rurale et la coopération France-Québec» (NC), 42, 4: 589-596

DONNEUR, André-Pierre, Joseph LEVITT, Pearson and Canada's Role in Nuclear Disarmament and Arms Control Negotiations, 1945-1957, 48, 1: 107-109; Denis SMITH, Diplomacy of
Fear - Canada and the Cold War 19411948, 43, 2: 277-279

DOSTALER, Gilles, Kari POLANYILEVITT, dir., The Life and Work of Karl Polanyi. A Celebration, 45, 4: 621-623

DOUVILLE, Éric, Yves POISSON, La naissance des Etats-Unis: des premiers colons au premier président (NB), 46, 3: 552

DROUIN, François, Jean-Pierre COLLIN, La cité coopérative canadiennefrançaise, 41, 1: 101-102; Danielle DION-McKINNON, Sillery. Au carrefour de l'histoire, 41, 4: 591-593

DRUMMOND, Anne, Margaret GILLETT et Ann BEER, dir., Our Own Agendas: Autobiographical Essays by Women Associated with McGill University, 50, 3: $450-452$

Du BERGER, Jean, Germain LEMIEUX, Les vieux m'ont conté, 30: Récits traditionnels, répertoire de Joseph Tremblay, 45, 1: 121-124

DUBÉ, Jean-Claude, Lorraine GADOURY, La noblesse en Nouvelle-France. Familles et alliances, 47, 3: 434-435

DUCHESNE, Raymond, Robert GAGNON; Armand J. ROSS, coll., Histoire de l'École polytechnique, 1873 1990. La montée des ingénieurs francophones, 46, 3: 523-526; Jacques MATHIEU, dir., Les dynamismes de la recherche au Québec, 46, 4: 688-690; Jacques SAINT-PIERRE, Les chercheurs de la mer. Les débuts de la recherche en océanographie et en biologie des pêches du Saint-Laurent, 48, 4: 584-586

DUCHESNEAU, Alain, Robert COMEAU, dir., Maurice Séguin, historien du pays québécois vu par ses contemporains, suivi de Les Normes de Maurice Séguin, 41, 4: 627-629

DUFOUR, Andrée, Louise ROY, Les Scurs de Sainte-Anne. Un siècle d'histoire, 2: 1900-1950, 47, 1: 138-140

DUMAIS, Monique, Jean RICHARD et Louis O'NEILL, dir., La question so- 
ciale hier et aujourd'hui. Colloque du centenaire de Rerum novarum, 48, 1 : 114-115

DUMAS, Monique, Louise GAGNON, L'apparition des modes enfantines au Québec, 47, 2: 276-278

DUMONT, François, Marcel FORTIN, Histoire d'une célébration. La réception critique immédiate d'Alain Grandbois, 1933-1963, 49, 1: 97-99

DUMONT, Micheline, Louise CHARPENTIER, René DUROCHER, Christian LAVILLE et Paul-André LINTEAU, Nouvelle histoire du Québec et du Cana$d a, 45,2$ : 251-256; Marta DANYLEWYCZ, Profession: religieuse. Un choix pour les Québécoises 1840-1920, 42, 4: 618-621; Claire GOURDEAU, Les délices de nos coeurs. Marie de l'Incarnation et ses pensionnaires amérindiennes, 1639-1672, 49, 2: 268-270; Yvan G. LEPAGE, Mémoires de Marie-Rose Girard, édition critique, 44, 3: 438-439; Denise ROBILLARD, Émilie TavernierGamelin, 43, 4: 597-599; Un bon manuel (NC), 45, 2: 251-256

DUPUIS, Jean-Claude, Robert COMEAU, Michel LÉVESQUE, Yves BÉLANGER et al., Daniel Johnson. Rêve d'égalité et projet d'indépendance, 45, 2: 270-272; Claude COUTURE, Le mythe de la modernisation du Québec. Des années 1930 à la Révolution tranquille, 46, 4: 678-679

DUTIL, Patrice, Hubert GUINDON, Quebec Society: Tradition, Modernity, and Nationhood, 43, 1: 113-114; Donald J. HORTON, André Laurendeau. La vie d'un nationaliste 1912-1968, 50, 2: 279-281

DUTIL, Patrice A., Réal BÉLANGER, Wilfrid Laurier: quand la politique devient passion, 41, 4: 613-616; Gilles GALLICHAN, Honoré Mercier. La politique et la culture, 48, 4: 555-557
$\mathbf{E}$

ECCL.ES, W. J., Jacques MATHIEU, $L a$ Nouvelle-France en Amérique du Nord, $X V I^{e}-X V I I I^{e}$ siècle, 46, 2: 333-334; Hubert WATELET, De France à Nouvelle-France: société fondatrice et société nouvelle, 49, 3: 445-446

EMERY, George, Don AKENSON, The Orangeman: the Life and Times of Ogle Gowan, 41, 2: 261-262

EWEN, Geoffrey, William KAPLAN, Everything That Floats: Pat Sullivan, Hai Banks, and the Seamen's Unions of Canada, 42, 2: 287-289

F

FAHMY-EID, Nadia, Marcel BELLAVANCE, Le Québec et la Confédération. Un choix libre? , 49, 3: 421-425

FARIBAULT-BEAUREGARD, Marthe, Marcel FOURNIER, Les Européens au Canada des origines à 1765 (NB), 46, 4: 707

FARKAS, Maryann, Johanne COLLIN, Changement d'ordonnance: mutations professionnelles, identité sociale et féminisation de la profession pharmaceutique au Québec, 1940-1980, 49, 4: 573-574

FECTEAU, Jean-Marie, Gérald BERNIER et Daniel SALEE, The Shaping of Québec Politics and Society. Colonialism, Power and the Transition to Capitalism in the 19th Century, 47, 2: 264-267; Bruce CURTIS, Building the Education State: Canada West, 18361871, 42, 2: 270-272; Laurier TURGEON, dir., Les productions symboliques du pouvoir, $X V I^{e}-X X^{e}$ siècle, 45,3 : 464-468; Yves VAILLANCOURT, 
L'évolution des politiques sociales au Québec, 1940-1960, 43, 1: 123-125

FERRETTI, Lucia, COLLECTIF CLIO, L'histoire des femmes au Québec depuis quatre siècles (NB), 46, 2: 367368; Jean HAMELIN, Histoire de l'Université Laval. Les péripéties d'une idée, 49, 4: 576-579; Giselle HUOT, Une femme au séminaire. Marie de la Charité (1852-1920) fondatrice de la première communauté dominicaine $d u$ Canada (1887), 41, 4: 603-604; Yvan LAMONDE, Combats libéraux au tournant du $X X^{e}$ siècle, 50, 2: 284-286; Roland LAMONTAGNE, Barrin de La Galissonière (NB), 46, 2: 373; Veronica STRONG-BOAG et Anita Clair FELLMAN, dir., Rethinking Canada. The Promise of Women's History, 45, 3: 472

FISHER, Lewis R., Shannon RYAN, Fish Out of Water: the Newfoundland Saltfish Trade, 1814-1914, 41, 2: 262264

FORTIN, Sarah, Kenneth McROBERTS, dir., Beyond Quebec. Taking Stock of Canada, 50, 2: 297-299

FOURNIER, Marcel, Stephen BROOKS et Alain-G. GAGNON, Les spécialistes des sciences sociales et la politique au Canada. Entre l'ordre des clercs et l'avant-garde, 48, 4: 544-548; Albert FAUCHER, dir., Cinquante ans de sciences sociales à l'Université Laval. L'histoire de la Faculté des sciences sociales (1938-1988), 42, 4: 624-626; Lucia FERRETTI, L'Université en réseau. Les 25 ans de l'Université du Québec, 49, 3: 427-430; Marlene SHORE, The Science of Social Redemption: McGill, the Chicago School, and the Origins of Social Research in Canada (NB), 42, 1: 127-128

FRANCIS, R. Douglas, Janet AJZENSTAT et Peter J. SMITH, dir., Canada's Origins. Liberal, Tory, or Republican?, 50, 2: 241-243

FRANK, David, Bryan D. PALMER, The Character of Class Struggle: Essays in
Canadian Working-Class History, 41, 2: 264-265

FRÉNETTE, Yves, Cahiers Charlevoix, 1: Études franco-ontariennes (NB), 50, 3: 483; Armand CHARTIER, Histoire des Franco-Américains de la NouvelleAngleterre, 1775-1990, 46, 2: 300-301; Chad GAFFIELD, dir., Histoire de l'Outaouais, 50, 1: 115-117; Yolande GRISÉ, dir., États généraux de la recherche sur la francophonie à l'extérieur du Québec, 50, 4: 617-619

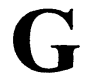

GADOURY, Lorraine, Françoise-Laure BURLET, Un rêve aristocratique en Nouvelle-France. La demeure de Charles Aubert de La Chesnaye, 50, 3: 437-438; Françoise DEROY-PINEAU, Madeleine de la Peltrie. Amazone du nouveau monde (NB), 47, 1: 147; François DOLLIER de CASSON, Histoire du Montréal (nouvelle édition critique par Marcel Trudel et Marie Baboyant) (NB), 47, 1: 147-148; textes établis et présentés par Pauline DUBÉ, La Nouvelle-France sous Joseph-Antoine Le Febvre de La Barre. Lettres, mémoires, instructions et ordonnances (16821685) (NB), 48, 2: 280-281; Geneviève RIBORDY, Les prénoms de nos ancêtres. Étude d'histoire sociale, 50, 2: 302-304; Marcel TRUDEL, Dictionnaire des esclaves et de leurs propriétaires au Canada français, 44, 4: 615616

GAFFIELD, Chad, Bruce S. ELLIOTT, Irish Migrants in the Canadas: a New Approach, 42, 2: 276-279; Richard M. REID, The Upper Ottawa Valley to 1855, 44, 4: 609-611

GAGNON, André, Gregory S. KEALEY et Reg WHITAKER, R.C.M.P. Security 
Bulletins: the Depression Years, Part I, 1933-1934 (NB), 48, 1: 119-120

GAGNON, France, Andrée FORTIN; Denys DELÂGE, Jean-Didier DUFOUR et Lynda FORTIN, coll., Histoires de familles et de réseaux. La sociabilité au Québec d'hier à demain, 43, 2: 262-264

GAGNON, François-Marc, Pierre BERTHIAUME et Émile LIZÉ, Foi et légendes. La peinture votive au Québec (1666-1945), 47, 1: 98-100; COLLECTIF, Un moment dans l'histoire. Vingt ans d'acquisition de peintures, de dessins et d'estampes aux Archives nationales du Canada, 46, 2: 305-307

GAGNON, Hervé, Richard GODBEER, The Devil's Dominion. Magic and Religion in Early New England, 46, 3: 533535; Cyril SIMARD; Andrée LAPOINTE et Corneliu KIRJAN, coll., Patrimoine muséologique au Québec. Repères chronologiques, 47, 3: 452453

GAGNON, Jean-Pierre, Yves BERNARD et Caroline BERGERON, Trop loin de Berlin. Des prisonniers allemands au Canada (1939-1946), 50, 2: 246-248; Terry COPP et Bill McANDREW, Battle Exhaustion: Soldiers and Psychiatrists in the Canadian Army, 1939-1945, 44, 4: 593-595; James W. ESSEX, Victory in the St. Lawrence. Canada's Unknown War, 41, 3: 440-442; Michael L. HADLEY, $U$ Boats Against Canada. German Submarines in Canadian Waters, 41, 3: 440-442; Réginald H. ROY, Débarquement et offensive des Canadiens en Normandie, 42, 2: 302-303

GAGNON, Nicole, COLLECTIF, Hommage à Marcel Rioux. Sociologie critique, création artistique et société contemporaine, 47, 1: 105-106; Lucia FERRETTI, Entre voisins. La société paroissiale en milieu urbain: SaintPierre-Apôtre de Montréal, 1848-1930, 46, 3: 519-521; Guy ROCHER, Entre les rêves et l'histoire, 43, 2: 275-277
GAGNON, Robert, Yves GINGRAS, L'origine de la recherche scientifique au Canada. Le cas des physiciens, 46, 2: 311-313; Pierre HARVEY, Histoire de l'École des Hautes études commerciales de Montréal, 1: 1887-1926, 49, 4: 579-581

GAGNON, Serge, Alain LABERGE, dir., Histoire de la Côte-du-Sud, 48, 2: 261265: Jean-Marc LAROUCHE, Éros et Thanatos sous l'oeil des nouveaux clercs, 46, 2: 326-329; Jean SIMARD, Les arts sacrés au Québec, 44, 3: 453455

GALLICHAN, Gilles, Jean De BONVILLE, dir., La presse québécoise de 1764 à 1914. Bibliographie analytique, 49, 4: 575-576; Jean-René LASSONDE, La Bibliothèque Saint-Sulpice, 1910-1931, 41, 2: 275-277

GARAND, Dominique, Pierre HÉBERT; Marie-Pier LUNEAU, coll., Lionel Groulx et L'appel de la race, 50, 3: 458-462

GAUDREAU, Guy, Ian RADFORTH, Bushworkers and Bosses: Logging in Northern Ontario, 1900-1980, 42, 2: 300-302; L. Anders SANDBERG, dir., Trouble in the Woods: Forest Policy and Social Conflict in Nova Scotia and New Brunswick, 46, 4: 701-703

GAUTHIER, Benoît, Robert SWEENY, Grace Laing HOGG et Richard RICE, Les relations ville/campagne: le cas du bois de chauffage, 43, 3: 430-431

GAUTHIER, Majella-J., Louis-Edmond HAMELIN, Le rang d'habitat: le réel et l'imaginaire, 48, 3: 439-441

GAUTHIER, Michelle, David KAREL, Dictionnaire des artistes de langue française en Amérique du Nord: peintres, sculpteurs, dessinateurs, graveurs, photographes et orfêvres (NB), 47, 2: 301

GAUTHIER, Raymonde, Claude BERGERON, L'architecture des églises du Québec, 1940-1985, 42, 1: 85-86; Christina CAMERON, Charles Baillairgé, Architect \& Engineer, 44, 3: 
428-430; François RÉMILLARD et Brian MERRETT, Demeures bourgeoises de Montréal, le Mille Carré doré 1850-1930, 43, 3: 423-425

GAUTHIER, Serge, Roger BLAIS, Jean Palardy. Peintre témoin de son époque (NB), 47, 1: 145-146

GÉLINAS, Isabelle, Yannick GASQUYRESCH, dir., Marseille-Montréal, centres culturels cosmopolites, 46, 3: 526528

GÉLINAS, Xavier, Léon BALCER, Léon Balcer raconte, 43, 1: 101-102; Marie BEAUPRÉ et Guy MASSICOTTE, Édouard Lacroix. Pionnier de l'entrepreneurship beauceron, 44, 1: 105-106; Léon DION, Le duel constitutionnel Québec-Canada (NB), 50, 3: 484-485; Gérard FILION, Fais ce que peux. En guise de mémoires, 43, 2: 260-262; Eugene FORSEY, A Life on the Fringe, 45, 2: 277-279; Les institutions politiques au Québec et au Canada (NB), 50, 2: 319

GENDRON, Mario, Paul BERNIER, Le cheval canadien, 46, 3: 501-503

GERVAIS, Gaétan, Robert CHOQUETTE, De la controverse à la concorde. L'Église d'Alexandria-Cornwall, 45, 3: 435-437

GINGRAS, Yves, Hélène-Andrée BIZIER, L'Université de Montréal. La quête du savoir, 48, 2: 243-245; Robert BOTHWELL, The History of Atomic Energy of Canada Limited, 42, 4: 602-605; Susan SHEETS-PYENSON, John William Dawson. Faith, Hope and Science, 50, 3: 474-476

GIRALDEAU, François, Annick GERMAIN et Jean-Claude MARSAN, dir., Aménager l'urbain, de Montréal à San Francisco. Politiques et design urbains, 42, 2: 285-287

GIRARD, André, André LEFEBVRE, Marie-Victorin, le poète éducateur, 41, 2: 290

GIROUARD, Guylaine, Line GOSSELIN, Les journalistes québécoises, 18801930, 50, 2: 274-277
GODIN, Jean Cléo, Pierre H. LEMIEUX, Nelligan amoureux, 46, 2: 361-364; Jacques MICHON, dir., Nelligan, 2: Poèmes et textes d'asile, 1900-1941, 46, 2: 361-364; Réjean ROBIDOUX et Paul WYCZYNSKI, dir., Nelligan, 1 : Poésies complètes, 1896-1941, 46, 2: 361-364; Paul WYCZYNSKI, dir., Émile Nelligan. Poèmes autographes, 46, 2: 361-364

Gossage, Peter, J. M. BUMSTED, dir., Interpreting Canada's Past, 1: Pre-Confederation; 2: Post-Confederation (NB), 48, 1: 118; Joanne BURGESS, Louise DECHÊNE, Paul-André LINTEAU et Jean-Claude ROBERT, dir.; Céline BOUCHARD, Rémi BOURDEAU, Michèle DAGENAIS et Lucy SICARD, coll., Clés pour l'histoire de Montréal. Bibliographie (NB), 47, 1: 146-147; J. I. LITTLE, The Child Letters: Public and Private Life in a Canadian Merchant-Politician's Family, 1841-1845, 49, 2: 274-275; Jean ROY et Daniel ROBERT, Le diocèse de Nicolet. Populations et territoires 1851-1991 (NB), 47, 1: 149

GOULET, Denis, Joseph HANAWAY et Richard CRUESS, McGill Medicine, 1: The First Half Century, 1829-1885, 50, 4: 619-621; Pierre MEUNIER, La chirurgie à l'Hôtel-Dieu de Montréal au XIX siècle, 44, 2: 274-276

GOULET, Henri, Pierre POULIN, Histoire du Mouvement Desjardins, 2: La percée des caisses populaires, 1920 1944, 50, 1: 129-131; réplique, 50, 4: 631-632; réponse de Henri GOULET, 50, 4: 633-635

GOURDEAU, Claire, Georges E. SIOUI, Les Wendats. Une civilisation méconnue, 49, 4: 592-594

GOW, James lain, Robert BRYCE, $M a$ turing in Hard Times: Canada's Department of Finance Through the Great Depression, 41, 2: 277-279

GRABOWSKI, Jan, C. M. BARBEAU, Mythologie huronne et wyandotte, 49, 2: 259-260; Alain BEAULIEU, Con- 
vertir les fils de Caïn. Jésuites et Amérindiens nomades en NouvelleFrance, 1632-1642, 45, 4: 595-596; Samuel de CHAMPLAIN; texte établi, présenté et annoté par Alain BEAULIEU et Réal OUELLET, Des Sauvages (NB), 48, 2: 280; Gilles THÉRIEN, dir., Figures de l'Indien, 50, 2: 309-312

GRANT, John Webster, Réal OUELLET, dir., Rhétorique et conquête missionnaire: le jésuite Paul LeJeune, 49, 2: $279-280$

GRATTON, Claude, Louis-Edmond HAMELIN, L'Obiou entre Dieu et Diable (NB), 45, 3: 471

GRENIER, Guy, Jacques BERNIER, $L a$ médecine au Québec. Naissance et évolution d'une profession, 43, 2: 253 255; André CELLARD, Histoire de la folie au Québec de 1600 à 1850, 45, 3: 433-435; Angus McLAREN, Our Own Master Race. Eugenics in Canada, 1885-1945, 45, 4: 612-614; André PARADIS et Hélène NAUBERT, Recension bibliographique: les maladies infectieuses dans les périodiques médicaux québécois du XIX siècle, 1: Matériaux pour l'histoire de la médecine québécoise, 42, 3: 481-482; François ROUSSEAU, La croix et le scalpel. Histoire des Augustines et de l'HôtelDieu de Québec, 2: 1892-1989, 49, 2: 285-287

GRIFFITHS, Naomi E. S, Alaric FAULKNER et Gretchen FEARSON-FAULKNER, Les Français à Pentagouet (1635-1674). Un portrait archéologique de la frontière acadienne, 44, 2 : 268-269

GROSMAIRE, Jean-Louis, Bernard PENISSON, Henri d'Hellencourt, un journaliste français au Manitoba (18981905), 41, 2: 266-267

GROULX, Patrice, Marcel MOUSSETTE, Le site du Palais de l'intendant à Québec. Genèse et structuration d'un lieu urbain, 50, 1: 127-129

GRUTMAN, Rainier, Maurice LEMIRE, Formation de l'imaginaire littéraire québécois (1764-1867), 48, 1: 106-107; Maurice LEMIRE, dir., La vie littéraire au Québec, 2: 1806-1839: le projet national des Canadiens, 47, 2: 283-285

GUÉRARD, François, Neville TERRY, The Royal Vic. The Story of Montreal's Royal Victoria Hospital 1894-1994, 48, 4: 588-590

\section{H}

HAMEL, Réginald, Réjean ROBIDOUX, Connaissance de Nelligan, 46, 4: 699701

HAMELLIN, Louis-Edmond, Jean-Claude BOIJLANGER, dir., Actes du XVI Congrès international des Sciences onomastiques. Le nom propre au carrefour des études humaines et des sciences sociales/Proper Names at the Crossroads of the Humanities and Social Sciences. Proceedings of the XVI International Congress of Onomastics Sciences, 45, 2: 263-265

HAME:LIN, Monique, collectif sous la direction de Anita CARON, Femmes et pouvoir dans l'Église, 46, 3: 510-512

HAMMOND, Lorne, Rowland LORIMER, dir., Conscience et survie. Écologie et culture au Canada (NB), 45, 4: 633

HANNA, David B., Marc H. CHOKO, Les grandes places publiques de Montréal, 43, 2: 257-258; COMMUNAUTÉ URBAINE DE MONTRÉAL, Les résidences, 41, 4: 605-607

HARDY, René, Diane SAINT-PIERRE et Yves HÉBERT, Archives paroissiales de la Côte-du-Sud: inventaire sommaire, 45, 1: 131-132

HARE, John, Jacques MICHON, dir., L'édition littéraire en quête d'autonomie. Albert Lévesque et son temps, 49, 4: $587-589$ 
HARE, John E., Maurice LEMIRE et al., La vie littéraire au Québec, 1: 17641805. La voix française des nouveaux sujets britanniques, 46, 4: 685-686

HEAP, Ruby, Terry CROWLEY, Agnes Macphail and the Politics of Equality, 45, 3: 437-440; A. B. McKILLOP, Matters of Mind. The University in Ontario, 1791-1951, 50, 2: 294-296; M'hammed MELLOUKI, Savoir enseignant et idéologie réformiste. La formation des maitres (1930-1964), 44, 1: 114-117; Lee STEWART, "It's Up to Yoù: Women at UBC in the Early Years, 44, 3: 455-458; Paul-André TURCOTTE, L'enseignement secondaire public des frères éducateurs (1920-1970). Utopie et modernité, 43, 1: $119-122$

HÉBERT, Léo-Paul, Lucien CAMPEAU, s.j., Catastrophe démographique sur les Grands Lacs (I), 41, 1: 79-81; Lucien CAMPEAU, s.j., Les premiers habitants du Québec (II), 41, 1: 79-81

HÉBERT, Pierre, Manon BRUNET et Serge GAGNON, Discours et pratiques de l'intime (NB), 48, 2: 279; Alain GRANDBOIS; édition critique par Chantal BOUCHARD et Nicole DESCHAMPS, Avant le chaos et autres nouvelles, édition critique, 46, 2: 315 316

HENLEY, Kevin, Ian M. DRUMMOND, Progress Without Planning: the Economic History of Ontario From Confederation to the Second World War, 42, 1: 96-98

HUBERT, Ollivier, Guy-Marie OURY, Monseigneur de Saint-Vallier et ses pauvres, 48, 1: 112-114

HUDON, François, François GUÉRARD, Histoire de la santé au Québec, 50, 3: 452-454

HUEL, Raymond, Pierre Alfred CHARLEBOIS, La vie de Louis Riel (NB), 46, 2: 366; SOCIÉTÉ HISTORIQUE DE SAINT-BONIFACE, Histoire de Saint-Boniface, 1: À l'ombre des cathé- drales. Des origines de la colonie jusqu'en 1870, 46, 2: 352-354

HYMAN, Harold, Georges-Émile LAPALME, Pour une politique. Le programme de la Révolution tranquille, 42, 2: 289-291

I

IGARTUA, José E, Donald KERR, Deryck W. HOLDSWORTH et PaulAndré LINTEAU, dir., Atlas historique du Canada, 3: Jusqu'au cour du $X X^{e}$ siècle, 45, 2: 284-287

IGARTUA, José E., Christopher ARMSTRONG et H. V. NELLES, Southern Exposure: Canadian Promoters in Latin America and the Caribeean 1896-1930, 43, 2: 247-250; Michael BLISS, Northern Enterprise: Five Centuries of $\mathrm{Ca}$ nadian Business, 41, 3: 419-422; Hubert CHARBONNEAU et al., Naissance d'une population. Les Français établis au Canada au XVIF siècle, 42, 3: 456459; Marthe FARIBAULT-BEAUREGARD, La vie aux Illinois au XVIII siècle. Souvenirs inédits de Marie-Anne Cerré. Un voyage de Montréal à Kamouraska en 1840, 42, 2: 279-281; Infodex: Index de la Presse (NB), 41, 3: 459; Fernand OUELLET, Economy, Class \& Nation in Quebec: Interpretive Essays (NB), 45, 4: 633-634

JACOBS, Ellen, Constance BACKHOUSE et David FLAHERTY, Challenging Times. The Women's Movement 
in Canada and the United States, 47, 3: 409-411

JANSON, Gilles, Bruce KIDD, The Struggle for Canadian Sport, 50, 4: 623-627; François ROUSSEAU, La croix et le scalpel. Histoire des Augustines et de l'Hôtel-Dieu de Québec (1639-1989), 1: 1639-1892, 44, 3: 446449

JARRELL, Richard A., Luc CHARTRAND, Raymond DUCHESNE et Yves GINGRAS, Histoire des sciences au Québec, 42, 4: 605-607; Yves GINGRAS, Pour l'avancement des sciences. Histoire de l'ACFAS, 19231993, 49, 1: 99-101; A. B. McKILLOP, Contours of Canadian Thought, 43, 3: 416-419; Michel TRÉPANIER, L'aventure de la fusion nucléaire: la politique de la Big Science au Canada, 50, 3: 476-478

JEAN, Dominique, Micheline DUMONT et Nadia FAHMY-EID, Les couventines. L'éducation des filles au Québec dans les congrégations religieuses enseignantes 1840-1960, 41, 4: 607-610; Denise LEMIEUX et Lucie MERCIER, Les femmes au tournant du siècle: 1880-1940. Ages de la vie, maternité et quotidien, 43, 4: 580-582; C. David NAYLOR, Private Practice. Public Payment. Canadian Medecine and the Politics of Health Insurance, 19111966, 41, 1: 99-101

JOHNSTON, Hugh, Kay J. ANDERSON, Vancouver's Chinatown. Racial Discourse in Canada, 1875-1980, 46, 4: 665-667

JOHNSTON, Wendy, J. M. BUMSTED, The Peoples of Canada: a Post-Confederation History, 47, 2: 269-271; Bruce CURTIS, True Government by Choice Men?: Inspection, Education and State Formation in Canada West, 46, 3: 514516; Ernest FORBES, dir., Les écoles du Nouveau-Brunswick: guide des sources archivistiques/New Brunswick Schools: a Guide to Archival Sources (NB), 46, 4: 706-707; Henry MILNER,
The Long Road to Reform: Restructuring Public Education in Quebec, 41, 1: 102-104; Christopher NICHOLL, Bishop's University, 1843-1970, 49, 2: 277-279

JONES, Richard, Paul AUBIN et LouisMarie CÔTÉ, Bibliographie de l'histoire du Québec et du Canada, 19461965 (NB), 41, 2: 287; Dorval BRUNELLE, Les trois colombes: essai, 41, 3: 446-447; LÉVESQUE, Michel et Robert COMEAU, dir.; Gilles BOURQUE, Roch DENIS, Paul-André LINTEAU, Michel LÉVESQUE, coll., Jean Lesage et l'éveil d'une nation, 43, 2: 258-260; Patricia DIRKS, The Failure of l'Action libérale nationale, 48, 1: 85-87; Gregory KEALEY et Reg WHITAKER, R.C.M.P. Security Bulletins: the War Series, 1939-1941, 43, 4: 578-579; Daniel LATOUCHE, Canada and Quebec. Past and Futur: an Essay, 42, 2: 291-292; Lucie LAURIN, Des luttes et des droits: antécédents et histoire de la Ligue des Droits de l'Homme de 1936 à 1975 (NB), 41, 4: 637-638; Michel LÉVESQUE; Rachel CASAUBON, coll., René Lévesque. Texies et entrevues 1960-1987, 46, 4: 686-688; David MacKENZIE, Inside the Atlantic Triangle: Canada and the Entrance of Newfoundland into Confederation 1939-1949, 41, 2: 280-281; Ian McKAY, The Challenge of Modernity: a Reader on Post-Confederation Canada, 47, 1: 131-133

JOYAL, André, Gilles PAQUET, dir., $L a$ pensée économique au Québec français: témoignages et perspectives, 45, 2: 2:88-291

JOYAL, Renée, Jean TRÉPANIER et Françoise TULKENS, Délinquance et protection de la jeunesse. Aux sources des lois belge et canadienne sur l'enfance, 50, 2: 313-315 
K

KASIRER, Nicholas, Nicolle FORGET, De la curatelle au curateur public, 50 ans de protection (NB), 50, 2: 321-322

KENNY, Stephen, John Herd THOMPSON et Stephen J. RANDALL, Canada and the United States. Ambivalent Allies, 49, 2: 291-293

KERMOAL, Nathalie, Franca IACOVETTA et Mariana VALVERDE, dir., Gender Conflicts. New Essays in Women's History (NB), 47, 3: 463-464

KOLISH, Evelyn, France PARENT, Entre le juridique et le social: le pouvoir des femmes à Québec au XVII siècle, 46, 2: 343-345; Réjean PELLETIER, Partis politiques et société québécoise: de Duplessis à Bourassa, 1944-1970, 43, 2: 271-273; Gordon T. STEWART, The Origins of Canadian Politics: a Comparative Approach (NB), 42, 1: 129-130; Brian YOUNG, The Politics of Codification. The Lower Canadian Civil Code of 1866, 49, 2: 293-295

$\mathbf{L}$

LABELLE, Ronald, Gary R. BUTLER, Histoire et traditions orales des Franco-Acadiens de Terre-Neuve, 50, 2: 250-253; Jean-Pierre PICHETTE, L'observance des conseils du maître, 46,2 : 346-348

LABERGE, Alain, J. F. BOSHER, Business and Religion in the Age of New France, 1600-1760: Twenty-Two Studies, 49, 4: 569-571; Gervais CARPIN, Histoire d'un mot: l'ethnonyme Canadien de 1535 à 1691 (NB), 50, 2: 319320; Sylvie DEPATIE, Mario LALANCETTE et Christian DESSU-
REAULT, Contributions à l'étude du régime seigneurial canadien, 41, 4: 597-599; Christiane PERRON, La vie d'un pionnier de l'ile d'Orléans. Robert Gagnon 1628-1703, 44, 4: 607-609

LABERGE, Danielle, Andrée LÉVESQUE, La norme et les déviantes. Des femmes au Québec pendant l'entredeux-guerres, 43, 2: 268-270

LACELLE, Claudette, Alain GELLY, Louise BRUNELLE-LAVOIE et Corneliu KIRJAN, La passion du patrimoine. La Commission des biens culturels du Québec, 1922-1994, 50, 2: 273 274

LACHANCE, André, D. Owen CARRIGAN, Crime and Punishment in Canada. A History, 46, 4: 674-676; Rémi CHENIER, Québec, ville coloniale française en Amérique: 1660-1690, 46, 2: 302-303; Phyllis LAMBERT et Alan STEWART, dir., Montréal, ville fortifiée au XVIII siècle, 47, 2: 281-283; Louis LAVALLÉE, La Pairie en Nouvelle-France, 1647-1760. Étude d'histoire sociale, 47, 3: 445-447; Rénald LESSARD, Se soigner au Canada aux XVII et XVII' siècles, 43, 4: 585-587

LACHANCE, Paul, Alfred Olivier HÉRO jr., La Louisiane et le Canada francophone, 1673-1989 (NB), 46, 3: 551

LACHAPELLE, Guy, Alain-G. GAGNON et Mary Beth MONTCALM, Québec: au-delà de la Révolution tranquille, 47, 3: 435-437

LACHAPELLE, Jacques, Madeleine FORGET, Les gratte-ciel de Montréal, 45, 2: 281-284; Raymonde GAUTHIER, La tradition en architecture québécoise: le $X X^{e}$ siècle, 45, 2: 281-284; Isabelle GOURNAY, dir., Ernest Cormier et l'Université de Montréal, 45, 2: 281-284

LACOURSIÈRE, Jacques, Gaston DESCHÊNES et Maurice PELLERIN, Le Parlement du Québec. Deux siècles d'histoire (NB), 46, 4: 705-706

LACROIX, Benoît, Serge GAGNON, Plaisir d'amour et crainte de Dieu. 
Sexualité et confession au Bas-Canada, 45, 2: 279-281

LAFOREST, Guy, André LAURENDEAU, Journal tenu pendant la Commission royale d'enquête sur le bilinguisme et le biculturalisme, 44, 3: 433435

LAFRANCE, Marc, James H. TAYLOR, Ottawa, an Illustrated History, 42, 1: 118-119

LAFRENIÈRE, Suzanne, Georges BUGNET, Albertaines, 45, 4: 598-600

LAGUEUX, Maurice, Jonathan GORMAN, Understanding History. An Introduction to Analytical Philosophy of History, 47, 3: 438-439

LAHAISE, Robert, Yolande GRISÉ et Jeanne d'Arc LORTIE, Les textes poétiques du Canada français, 1606-1867, 6: 1856-1858, 47, 4: 556-559; Yolande GRISÉ et Jeanne d'Arc LORTIE, Les textes poétiques du Canada français, 1606-1867, 7: 1859, 49, 3: 433-436; Yolande GRISÉ et Jeanne d'Arc LORTIE, Les textes poétiques du Canada français, 1606-1867, 8: 1860 (NB), 49, 4: 600-601; Yolande GRISÉ et Jeanne d'Arc LORTIE, Les textes poétiques $d u$ Canada français, 1606-1867, 9: 18611862 (NB), 50, 2: 323; Jacques MATHIEU et Jacques LACOURSIÈRE, Les Mémoires québécoises, 46, 4: 690692; Mission Montréal. Les congrégations religieuses dans l'histoire de la ville (NB), 47, 2: 302; John R. PORTER et Jean BÉLISLE, La sculpture ancienne au Québec - Trois siècles d'art religieux et profane, 41, 2: 246249; Les prêtres de Saint-Sulpice au Canada, 47, 2: 297-298

LAJEUNESSE, Marcel, Pierre RAJOTTE, Les mots du pouvoir ou le pouvoir des mots. Essai d'analyse des stratégies discursives ultramontaines au XIX siècle, 46, 4: 694-696

LALANCETTE, Mario, René JETTÉ, Traité de généalogie (NB), 46, 4: 707709; Pierre LAMBERT, Les origines de Beloil (NB), 46, 2: 370-371; Yves
LANDRY, dir., Pour le Christ et le Roi: la vie au temps des premiers Montréalais, 47, 4: 564-566; Jean PARISEAU et Serge BERNIER, Les Canadiens français' et le bilinguisme dans les Forces armées canadiennes, 1: 1763-1969: Le spectre d'une armée bicéphale, 42, 2: 296-300; Jean-Pierre PROULX, La pêche de la baleine dans l'Atlantique Nord jusqu'au milieu du XIX $X^{e}$ siècle, 41, 3: 416-417; Jean-Paul-Médéric TREMBLAY, Tout un été de guerre. La conquête anglaise vue de la Baie Saint-Paul, 1735-1785, 41, 2: 288

LAMARRE, Jean, Gérard J. BRAULT, The French-Canadian Heritage in New England, 43, 2: 255-257; Maurice SÉGUIN, Une histoire du Québec. Vision d'un prophète (NB), 50, 2: 324

LAMONDE, Yvan, Alan F. J ARTIBISE, dir., Interdisciplinary Approaches to Canadian Society. A Guide to the Literature, 44, 3: 420-421; André BEAULIEU, Jean HAMELIN et al., La presse québécoise, des origines à nos jours, 44, 3: 427-428; Michel BELLEFLEUR, L'Église et le loisir au Québec avant la Révolution tranquille (NB), 41, 3: 458-459; Arthur BUIES, Correspondance (1855-1901), édition critique par Francis PARMENTIER, 47, 1: 103-105; Arthur BUIES, Chroniques II, édition critique par Francis PARMENTIER, 46, 3: 508-510; Léon DION, Québec, 1945-2000, 2: Les intellectuels et le temps de Duplessis, 47, 3: 424-426; Andrée FORTIN, Passage de la modernité. Les intellectuels québécois et leurs revues, 48, 1: 87-90; Louis FRÉCHETTE, Satires et polémiques ou l'École cléricale au Canada, édition critique par Jacques BLAIS, Guy CHAMPAGNE et Luc BOUVIËR, 47, 3: 430-433; François GALLAYS, Sylvain SIMARD et Paul WYCZYNSKI, Le Nigog, 42, 2: 283285; Giselle HUOT, Juliette LALONDE-RÉMILLARD et Pierre TRÉPANIER, Correspondance de Lionel 
Groulx, 1894-1967, 2: Un étudiant à l'école de l'Europe, 1906-1909, édition critique, 47, 4: 559-561; Robert HÉBERT, Le procès Guibord ou l'interprétation des restes, 46, 4: 681-683

LAMOUREUX, Diane, Jocelyne LAMOUREUX, Michèle GÉLINAS et Katy TARI, Femmes en mouvement. Trajectoires de l'Association féminine d'éducation et d'action sociale, 48, 1: 98-100; Mariette SINEAU et Évelyne TARDY, Droits des femmes en France et au Québec, 49, 1: 105-108

LAMY, Jean-Paul, Yvan G. LEPAGE et Germaine GUÈVREMONT, Le Survenant, édition critique, 43, 4: 583-585; RINGUET; édition critique par Jean PANNETON, Roméo ARBOUR, et Jean-Louis MAJOR, Trente arpents, édition critique, 46, 4: 697-699

LANDRY, Kenneth, Mary Lu MacDONALD, Literature and Society in the Canadas, 1817-1850, 48, 2: 267-269

LANDRY, Nicolas, Histoires de pêche (NC), 46, 2: 273-284; Colin HOWELL et Richard TWOMEY, dir., Jack Tar in History: Essays in the History of Maritime Life and Labour, 46, 2: 322-324

LANDRY, Yves, Micheline D'ALLAIRE, Les dots des religieuses au Canada français, 1639-1800, 41, 2: 253-256; Danielle GAUVREAU, Québec. Une ville et sa population au temps de la Nouvelle-France, 46, 3: 528-530; Marcel TRUDEL, La population du Canada en 1666. Recensement reconstitué (NB), 50, 2: 325

LANGLOIS, Simon, Alain-G. GAGNON, Québec: État et société, 50, 1: 117-120

LANTHIER, Pierre, Gérald BERNIER, Robert BOILY et Daniel SALÉE, Le Québec en chiffres de 1850 à nos jours, 42, 3: 445-447; Jean-Pierre CHARLAND, Les pâtes et papiers au Québec, 1880-1980: technologies, travail et travailleurs, 44, 3: 430-433; Camil GIRARD et Normand PERRON, Histoire du Saguenay-Lac-Saint-Jean, 44,
2: 270-271; Francess G. HALPENNY et Jean HAMELIN, dir., Dictionnaire biographique du Canada, 12: de 1891 à 1900, 44, 4: 597-599; José E. IGARTUA, Arvida au Saguenay: naissance d'une ville industrielle, 50, 4: 621-623; Fabien LAROCHELLE, Histoire de Shawinigan (NB), 43, 1: 128-129; J. Rodney MILLARD, The Master Spirit of the Age: Canadian Engineers and the Politics of Professionalism, 43, 3: 421-423; Jacques ROUILLARD, dir., Guide d'histoire du Québec du Régime français à nos jours. Bibliographie commentée, 49, 3: 440-443; Carl M. WALLACE et Ashley THOMSON, Sudbury: Rail Town to Regional Capital, 49, 1: 110-113; Brian YOUNG, In Its Corporate Capacity: the Seminary of Montreal as a Business Institution, 1816-1876, 41, 2: 258-261

LAPERRIÈRE, Guy, Robert CHOQUETTE, La foi gardienne de la langue en Ontario, 1900-1950, 41, 4: 620621; Marie-Aimée CLICHE, Les pratiques de dévotion en Nouvelle-France. Comportements populaires et encadrement ecclésial dans le gouvernement de Québec, 42, 3: 459-461; L'histoire religieuse du Québec: principaux courants, 1978-1988 (NC), 42, 4: 563-578; Yves RAGUIN, s.j., Au-delà de son rêve: Délia Tétreault, 46, 3: 541-543; Nive VOISINE, Les Frères des Écoles chrétiennes au Canada, 1: La conquête de l'Amérique, 1837-1880, 42, 2: 310-312

LAPOINTE, Simon, Pierre GODIN, René Lévesque: un enfant du siècle, 49, 2: 267-268

LAROCQUE, Paul, Jean-François CAR$\mathrm{DIN}$, La crise d'octobre 1970 et le mouvement syndical québécois, 42, 3: 450-452; Jean-Charles FORTIN, Antonio LECHASSEUR et al., Histoire du Bas Saint-Laurent, 48, 3: 435-437; Odette VINCENT, dir., Histoire de l'Abitibi-Témiscamingue, 50, 2: 317 318 
LaROSE, André, Serge COURVILLE; Jacques CROCHETIÈRE, Philippe DESAULNIERS et Johanne NOËL, coll., Paroisses et municipalités de la région de Montréal au XIX $X^{e}$ siècle (1825-1861). Répertoire documentaire et cartographique, 42, 4: 610-611; Serge COURVILLE et Serge LABRECQUE; Jacques FORTIN, coll., Seigneuries et fiefs du Québec: nomenclature et cartographie, 42, 4: 611-615; Marthe FARIBAULT-BEAUREGARD et Ève BEAUREGARD-MALAK, $L a$ généalogie. Retrouver ses ancêtres, 42 , 2: 282; Françoise NOËL, The Christie Seigneuries. Estate Management and Settlement in the Upper Richelieu Valley, 1760-1854, 47, 1: 133-135

LARUE, Jean-Marie, Chantal HÉBERT, Le Burlesque québécois et américain, 43, 2: $267-268$

LaRUE, Richard, F. Murray GREENWOOD, Legacies of Fear. Law and Politics in Quebec in the Era of the French Revolution, 48, 3: 437-439; Brendan O'BRIEN, Speedy Justice. The Tragic Last Voyage of His Majesty's Vessel Speedy, 47, 1: 135-136

LATOUR, Marie-Josée, Jean Du BERGER et Jacques MATHIEU, Les ouvrières de Dominion Corset à Québec (1886-1988) (NB), 48, 4: 591

LAUGRAND, Frédéric, Robert CHOQUETTE, The Oblate Assault on Canada's Northwest, 49, 4: 571-572

LAUZON, Gilles, Denis GRAVEL, Histoire du Village des Rapides - Un quartier de LaSalle, 48, 1: 92-94

LAVERDURE, Paul, Raymond HUEL, dir., Études oblates de l'Ouest 1. Actes $d u$ premier colloque sur l'histoire des Oblats dans l'Ouest et le Nord canadien/Western Oblate Studies 1. Proceedings of the First Symposium on the History of the Oblates in Western and Northern Canada, 44, 4: 599-601; Tendances dominantes de l'historiographie religieuse au Canada anglais, 1979. 1988 (NC), 42, 4: 579-587; Robert
WRIGHT, $A$ World Mission: Canadian Protestantism and the Quest for a New International Order, 1918-1939, 46, 3: 547-549

LAVOIE, Elzéar, Marc RABOY, Missed Opportunities. The Story of Canada's Broadcasting Policy, 44, 3: 441-443

LEBEL, Jean-Marie, Denis MONIÈRE, Ludger Duvernay et la révolution intellectuelle au Bas-Canada, 42, 2: 292295

LEBEL, Maurice, Axel MAUGEY, Le roman de la francophonie, 48, 2: 269271

LeBEUF, Marcel-Eugène, Jacques LAPLANTE, Prison et ordre social au Québec, 44, 4: 601-603

LeBLANC, Phyllis E., Ernest R. FORBES, Challenging the Regional Stereotype: Essays on the 20th Century Maritimes, 44, 1: 108-111

LECHASSEUR, Antonio, Martine CARDIN, Archivistique: information, organisation, mémoire. L'exemple du Mouvement coopératif Desjardins, 1900 1990, 50, 3: 440-443

LEDOYEN, Alberte, Julien BAUER, Les minorités au Québec, 49, 2: 260-262

LEFEBVRE, Marie-Thérèse, Élisabeth GALLAT-MORIN, Un manuscrit de musique française classique. Étude critique et historique. Le Livre d'Orgue de Montréal, 42, 3: 467-469

LEFEBVRE, Solange, Raymond LEMIEUX et Micheline MILOT, dir., Les croyances des Québécois. Esquisses pour une approche empirique, 48, 1 : $102-104$

LÉGARÉ Jacques, COLLECTIF, Femmes et questions démographiques: un nouveau regard, 46, 4: 676-678

LEGAULT, Chantal, Yolande GRISÉ et Jeanne d'Arc LORTIE, Les textes poétiques du Canada français, 1606-1867, 4: 1838-1849, 46, 2: 320-322; réplique, 47, 3: 465-468; Yolande GRISÉ et Jeanne d'Arc LORTIE, Les textes poétiques du Canada français, 1606-1867, 5: 1850-1855 (NB), 47, 1: 148-149; Jo- 
seph MELANÇON, dir., Les métaphores de la culture, 47, 3: 447-449

LEGAULT, Roch, André CHARBONNEAU, Les fortifications de l'île aux Noix: reflet de la stratégie défensive sur la frontière du Haut-Richelieu aux $X V I I I^{e}$ et $X I X^{e}$ siècles, 48, 4: 551-552; Ernest CLARKE, Siege of Fort Cumberland, 1776. An Episode in the American Revolution, 50, 2: 253-255; Richard A. PRESTON, Au service du Canada. Histoire du Royal Military College depuis la Deuxième Guerre mondiale, 47, 2: 295-297

LEMIEUX, Denise, Denyse BAILLARGEON, Ménagères au temps de la Crise, 46, 2: 285-287; Madeleine FERRON, Adrienne. Une saga familiale, 48, 2: 252-253; B. JEWSIEWICKI, dir., Récits de vie et Mémoires, vers une anthropologie du souvenir, 42 , 3: 472-474; Peter WARD, Courtship, Love and Marriage in Nineteenth Century English Canada, 44, 2: 284286; John E. ZUCCHI, The Little Slaves of the Harp. Italian Child Street Musicians in Nineteenth-Century Paris, London, and New York, 47, 3: 456-458

LEMIEUX, Lucien, Noël BAILLARGEON, Le Séminaire de Québec de 1800 à 1850, 49, 3: 419-421; Denise ROBILLARD, Paul-Émile Léger. Évolution de sa pensée, 1950-1967, 48, 3: 449-451

LEMIEUX, Raymond, Joan MARSHALL, A Solitary Pillar. Montreal's Anglican Church and the Quiet Revolution, 49, 2: 275-277; Madeleine SAUVÉ, L'Institut supérieur de sciences religieuses de la Faculté de théologie, 49, 4: 591-592; Paul-André TURCOTTE, Intransigeance ou compromis. Sociologie et histoire du catholicisme actuel, 49, 4: 594-596

LEMIEUX, Vincent, Roger OUELLETTE, Le Parti acadien. De la fondation à la disparition, 1972-1982 (NB), 47, 2: 302-303
LEMOINE, Réjean, Michel L'HÉBREUX, Une merveille $d u$ monde: le pont de Québec, 41, 2: 289

LÉONARD, Jacques, Les Cahiers des Dix, $n^{\circ}$ 44, 45, 1: 111-112; Les Cahiers des Dix, $n^{\circ} 45,45,1$ : 111-112; LouisArmand de Lom d'Arce, baron de LAHONTAN; édition critique par Réal OUELLET; Alain BEAULIEU, coll., Euvres complètes, édition critique, 45 , 3: 445-448; André VACHON, Ramas I + Histoire du Canada, 45, 2: 294-296; André VACHON, Ramas II + Propos sur l'histoire, la recherche et la publication, la patrie et le patrimoine, 45, 2: 294-296

LEPAGE, André, Rosemary E. OMMER, From Outpost to Outport. A Structural Analysis of the Jersey-Gaspé Cod Fishery, 1767-1886, 45, 4: 617-621

LÉPINE, Luc, Mary Beacock FRYER, Volunteers \& Redcoats, Raiders \& Rebels. A Military History of the Rebellions in Upper Canada, 42, 2: 282-283; William GRAY, Soldiers of the King. The Uper Canadian Militia, 18121815: a Reference Guide (NB), 50, 4: 637-638

LEROUX, Serge, Sylvain SIMARD, dir., La Révolution française au Canada français, 46, 4: 703-705

LESSARD, Rénald, Jean-René BRETON et Andrée RAICHE-DUSSAULT, $B i$ bliographie de Beauce-Etchemin, 47, 4: 551-552; Denis GOULET et André PARADIS, Trois siècles d'histoire médicale au Québec. Chronologie des institutions et des pratiques, 16391939, 47, 4: 555-556

LÉTOURNEAU, Jocelyn, Craig BROWN, dir., Histoire générale du Canada, 43, 1: 104-107; Fernand DUMONT, Genèse de la société québécoise, 50, 1: 110-115; Yvan LAMONDE; Gérard PELLETIER, coll., Cité libre. Une anthologie (NB), 46, 2: 371-372; réplique, 46, 3:556-557; réponse de Jocelyn LÉTOURNEAU, 46, 3: 557; Vincent LEMIEUX, Les relations de pouvoir dans 
les lois. Comparaison entre les gouvernements du Québec de 1944 à 1985, 46, 4: 683-685

LEVASSEUR, Roger, Jean DAIGLE, Une force qui nous appartient. La Fédération des caisses populaires acadiennes, 1936-1986, 44, 2: 265-266; Jacques-T. GODBOUT, La démocratie des usagers, 41, 3: 450-453

LEVER, Yves, Roland COSANDEY, André GAUDREAULT et Tom GUNNING, dir., Une invention du diable? Cinéma des premiers temps et religion/An Invention of the Devil? Religion and Early Cinema, 47, 1: 108-110

LÉVESQUE, Andrée, Gregory S. KEALEY et Reg WHITAKER, dir., R.C.M.P. Security Bulletins. The War Series, Part II, 1942-45, 47, 3: 442-443

LÉVESQUE, Michel, Jean-Guy GENEST, Godbout (NB), 50, 4: 635-636; Denis MONIÈRE, Le combat des chefs. Analyse des débats télévisés au Cana$d a, 47,3: 449-452$

LEVINE, Marc V., Paul-André LINTEAU, Histoire de Montréal depuis la Confédération, 47, 2: 286-288

LIMOGES, Camille, Ronald L. NUMBERS, dir., Medicine in the New World. New Spain, New France, and New England, 41, 3: 413-416; Suzanne ZELLER, Inventing Canada. Early Victorian Science and the Idea of a Transcontinental Nation, 42, 1: 121124

LITTLE, J. I., Serge LAURIN, Histoire des Laurentides, 44, 1: 112-114; Françoise NOËL, Competing for Souls: Missionary Activity and Settlement in the Eastern Townships, 1784-1851, 42, 3: 479-481; Brian YOUNG et John A. DICKINSON, A Short History of Quebec: a Socio-Economic Perspective, 43, 1: $125-127$

LORD, France, Anne-Hélène KERBIRIOU, Les Indiens de l'Ouest canadien vus par les Oblats, 50, 3: 462-464
M

MAIRE, Claude, J. F. BOSHER, The $\mathrm{Ca}$ nada Merchants, 1713-1763, 42, 2: 263-266

MALOUIN, Marie-Paule, Micheline DUMONT, Les religieuses sont-elles féministes?, 50, 2: 266-267; Myriam SIMA.RD, L'enseignement privé. 30 ans de débats, 48, 2: 277-278

MARCOTTE, Gilles, Gérard BERGERON, Lire François-Xavier Garneau, 1809-1866, "historien national», 49, 3 : 425-427

MARCOUX, Yves, Helen HORNBECKTANNER, dir., Atlas of Great Lakes Indian History, 41, 3: 412-413

MARSAN, Jean-Claude, France GAGNON-PRATTE, Maisons de campagne des Montréalais 1892-1924. L'architecture des frères Maxwell, 42, 3: 465-467

MARSHALL, Dominique, Jean-Pierre CHARLAND et Mario DÉSAUTELS, Système technique et bonheur domestique. Rémunération, consommation et pauvreté au Québec, 1920-1960, 47, 3: 418-421; Gaston DESJARDINS, L'amour en patience. La sexualité adolescente au Québec, 1940-1960, 50, 2: 263-266

MARTEL, Gilles, George F. G. STANLEY, Toil and Trouble. Military Expeditions to Red River, 44, 1: 119-121

MARTEL, Marcel, Michel DOUCET, Le discours confisqué, 50, 3: 448-450

MARTIJN, Charles A, Ruth Holmes WHITEHEAD, The Old Man Told Us. Excerpts from Micmac History, 1500 1950, 46, 2: 359-360

MARTIN, Paul-Louis, Bernard AUDET, Avoir feu et lieu dans l'íle d'Orléans au XVII siècle, 44, 3: 421-424; Germain CA.SAVANT, dir., Les appartements (NB), 46, 2: 364-365; Jacques GUIMONT, La Petite-Ferme du cap Tour- 
mente, un établissement agricole tricentenaire. De la ferme de Champlain aux grandes volées d'oies, 50, 3: 454456

MASSE, Georges, Marc DESJARDINS, Bibliographie des Îles-de-la-Madeleine, 42, 3: 461-463; Serge GAUDREAU, Au fil du temps. Histoire de l'industrie textile à Magog (1883-1993), 50, 2: 269-272

MASSICOTTE, Daniel, André LACHANCE, La vie urbaine en NouvelleFrance, 41, 4: 595-597

MATHIEU, Jacques, $\dot{A}$ propos de la publication de Alice Jean E. Lunn, «Développement économique de la Nouvelle-France, 1713-1760». Les temps de l'histoire au Québec (NC), 41, 1: 7175; Gabrielle LACHANCE, dir., Mémoire d'une époque. Un fonds d'archives orales au Québec, 41, 3: 453-456; Montréal citée. 60 ans d'histoire à travers les archives sonores (1930-1990), 46, 4: 692-694

MAYER, Francine M., Gérard BOUCHARD et Marc DeBRAEKELEER, Histoire d'un génôme. Population et génétique dans l'est du Québec, 46, 3: 503-506

McLAUGHLIN, Kenneth, Chad GAFFIELD, Language, Schooling, and Cultural Conflict. The Origins of the French-Language Controversy in Ontario, 42, 3: 463-465

McNEIL, Derick, Éphrem BOUDREAU, Glossaire du vieux parler acadien, 47, 2: 267-269; Léopold LANCTOT, o.m.i., L'Acadie des origines, 16031771, 47, 2: 267-269; Clarence LEBRETON, Le "Caraquet Flyer», 47, 2: 267-269; Clarence LEBRETON, Le Collège de Caraquet, 47, 2: 267-269; Yvon LEGER, L'Acadie de mes ancêtres, 47, 2: 267-269

McNICOLL, Claire, CAHIERS DE GÉOGRAPHIE DU QUÉBEC, La géographie du Québec cinquante ans après Raoul Blanchard, 42, 1: 87-89
MELANÇON, François, Aurélien BOIVIN, Gilles DORION et Kenneth LANDRY, dir., Questions d'histoire littéraire. Mélanges offerts à Maurice Lemire, 50, 2: 248-250; François-Marc GAGNON, Images du castor canadien, $X V I^{e}-X V I I I^{e}$ siècles, 49, 2: 265-267

MELANÇON, Joseph, Francophonies d'Amérique, 47, 3: 428-430

MICHEL, Louis, Serge COURVILLE, Entre ville et campagne. L'essor $d u$ village dans les seigneuries du BasCanada, 45, 2: 274-277

MICHON, Jacques, Raymond BRODEUR, dir.; Brigitte CAULIER, Bernard PLONGERON, Jean-Paul ROULEAU et Nive VOISINE, coll., Les catéchismes au Québec, 1702-1963, 45, 2: 265-268; Henri TRANQUILLE, Lettres dangereuses à Yves Beauchemin (NB), 46, 2: 375

MILleR, Carman, A Heritage At Risk: the Canadian Militia As a Social Institution, 43, 3: 438-440; Desmond MORTON, Une histoire militaire $d u$ Canada, 1608-1991, 47, 4: 571-573; Desmond MORTON, Silent Battle. Canadian Prisoners of War in Germany, 1914-1919, 47, 4: 571-573; Desmond MORTON et Glenn WRIGHT, Winning the Second Battle: Canadian Veterans and the Return to Civilian Life 1915-1930, 42, 2: 295-296; Peter NEARY, Newfoundland in the North Atlantic World, 1929-1949, 44, 2: 279 281

MIMEAULT, Mario, Olivier GUYOTJEANNIN, Saint-Pierre et Miquelon, 41, 4: 593-595; Nicolas LANDRY, Les pêches dans la péninsule acadienne 1850-1900, 50, 2: 286-287

MONIĖRE, Denis, Louis BALTHAZAR, Guy LAFOREST et Vincent LEMIEUX, dir., Le Québec et la restructuration du Canada, 1980-1992: enjeux et perspectives, 46, 2: 287-289; Anne LÉGARÉ, La souveraineté est-elle dépassée? , 47, 1: 120-122 
MOREAU, Jean-François, Olive Patricia DICKASON, Le mythe du Sauvage, 48, 2: 249-252

MORIN, Michel, Evelyn KOLISH, Nationalismes et conflits de droits: le débat du droit privé au Québec, 1760-1840, 49, 2: 271-273

MORTON, Desmond, John F. CONWAY, Des comptes à rendre. Le Canada anglais et le Québec, de la Conquête à l'Accord de Charlottetown, 50, 2: 257-259; Jean-Pierre GAGNON, Le $22^{e}$ bataillon (canadien-français) $1914-$ 1919: étude socio-militaire, 41, 2: 273 275

MOUSSETTE, Marcel, Pierre BEAUDET, dir., Les dessous de la terrasse à Québec: archéologie dans la cour et les jardins du Château Saint-Louis, 44, 3: 424-426

MURRAY, Jocelyne, Léo-Paul HÉBERT, Le rôle socio-économique du Collège de Joliette (1846-1991) (NB), 47, 3: 463; Alison PRENTICE et Marjorie R. THEOBALD, Women Who Taught. Perspectives on the History of Women and Teaching, 45, 3: 460-461; Denise ROBILLARD, La traversée du Saguenay. Cent ans d'éducation. Les Scurs de Notre-Dame du Bon-Conseil de Chicoutimi, 1894-1994, 49, 2: 281-283

$\mathbf{N}$

NADEAU, Yves, Patrick BRODE, The Odyssey of John Anderson, 44, 4: 589591

NAHUET, Robert, Normand FORTIER, Guide des fonds d'histoire orale au Canada/Guide to Oral History Collections in Canada, 48, 4: 553-554

NEATBY, Nicole, Paul AXELROD, $M a-$ king a Middle Class: Student Life in English Canada During the Thirties,
45, 2: 259-261; Paul AXELROD et John G. REID, dir., Youth, University, and Canadian Society. Essays in the Social History of Higher Education, 43, 2: 251-253

NOËL. Françoise, Sidney Thomson FISHER, The Merchant-Millers of the Humber Valley: a Study of the Early Economy of Canada, 41, 1: 81-83; J. I. LITTLE, Nationalism, Capitalism, and Colonization in Nineteenth-Century Quebec. The Upper St. Francis District, 43, 4: 590-591

NORE:AU, Pierre, René DIONNE, $L a$ littérature régionale aux confins de l'histoire et de la géographie, 48, 2: 259-261; Guy GAUDREAU, dir., $D u$ centre des jeunes au Carrefour francophone, 1951-1990. Quarante ans de vie communautaire et culturelle à Sudbury, 48, 2: 259-261; Lauren McKINSEY, Victor KONRAD et al., Une frontière dans la tête: culture, institutions et imaginaire canadiens, 46, 2: 334-337

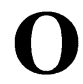

OLSCAMP, Marcel, Maurice LEMIRE, La littérature québécoise en projet au milieu du XIX siècle, 49, 1: 103-105

ORBAN, Edmond, Thierry HENTSCH, Introduction aux fondements du politique, 48, 1: 94-95

OUELLET, Fernand, Chad GAFFIELD, Aux origines de l'identité franco-ontarienne. Éducation, culture, économie, 48, 2: 253-257

OUELLETTE, Françoise-Romaine, Gérard BOUCHARD et Martine SEGALEN, dir., Dynamiques culturelles interrégionales au Québec et en France. Construction d'une enquête, 50, 4: 615617; Serge GAGNON, Mariage et fa- 
mille au temps de Papineau, 48, 2: 257259

OWRAM, Doug, Thomas P. SOCKNAT, Witness Against War. Pacifism in Canada 1900-1945, 41, 4: 617-619

$\mathbf{P}$

PACOM, Diane, Jocelyn LÉTOURNEAU, dir.; Roger BERNARD, coll., La question identitaire au Canada francophone: récits, parcours, enjeux, hors-lieux, 48, 3: 447-449; Serge WAGNER et Pierre GRENIER, Analphabétisme de minorité et alphabétisation d'affirmation nationale, à propos de l'Ontario français, 45, 4: 626-629

PAQUET, Martin, Charles CASTONGUAY, L'assimilation linguistique: mesure et évolution, 1971-1986 (NB), 49, 2: 301; Marcel CÔTÉ et Gaston DUSSAULT; Richard DERY et Pierre SIMARD, coll., Claude Castonguay. Architecte social et gestionnaire (NB), 49, 2: 302; Simon LANGLOIS et Yves MARTIN, dir., L'horizon de la culture. Hommage à Fernand Dumont, 50, 2: 288-290; Paul-André LINTEAU, Histoire du Canada (NB), 48, 4: 593; Dick SPENCER, Trumpets and Drums. John Diefenbaker on the Campaign Trail, 48, 4: 586-588

PAQUETTE, Jean-Marcel, Pierre BERTHIAUME, L'aventure américaine au XVIII siècle: $d u$ voyage à l'écriture (NB), 46, 3: 550

PARADIS, André, Marcel FOURNIER, Yves GINGRAS et Othmar KEEL, Sciences et médecine au Québec, perspectives sociohistoriques, "L'histoire de la médecine: une porte ouverte sur l'histoire sociale» (NC), 42, 1: 73-83; Wendy MITCHINSON, The Nature of Their Bodies: Women and Their Doc- tors in Victorian Canada, 46, 2: $337-$ 340; La naissance de la psychanalyse à Montréal, 41, 3: 443-446

PARENT, Colette, Andrée LÉVESQUE, Résistance et transgression. Études en histoire des femmes au Québec, 50, 3: 464-466

PARISEAU, Jean, Pierre BLAIS, Loup solitaire. Un mercenaire québécois pleure le Viêt-Nam (NB), 45, 4: 629630; Sandra GWYN, Tapestry of War: a Private View of Canadians in the Great War, 49, 4: 596-598; Walter HILDEBRANDT, La bataille de Batoche. Une petite guerre britannique contre des Métis retranchés, 41, 1: 9293; Robert PAINCHAUD, Un rêve français dans le peuplement de la Prairie, 41, 1: 87-89; Robert A. STEBBINS, The Franco-Calgarians: French Language, Leisure and Linguistic LifeStyle in an Anglophone City (NB), 49, 2: 302-303; Brian Loring VILLA, Unauthorized Action: Mountbatten and the Dieppe Raid, 46, 3: 546-547; Jeffery WILLIAMS, First in the Field: Gault of the Patricias, 49, 4: 596-598

PARMENTIER, Francis, Claude GALARNEAU et Maurice LEMIRE, dir., Livre et lecture au Québec (18001850), 43, 1: 108-112

PAYMENT, Diane, D. N. SPRAGUE, Canada and the Metis, 1869-1885, 43, 3: $428-430$

PAYMENT, Diane Paulette, Frits PANNEKOEK, Snug Little Flock. The Social Origins of the Riel Resistance of 1869-70, 46, 2: 341-343

PELletier, Louis, Micheline D'ALLAIRE, Les dots des religieuses au Canada français, 1639-1800, 41, 2: 253-256

PELLETIER, Réjean, John C. COURTNEY, Do Conventions Matter? Choosing National Party Leaders in Cana$d a, 50,2$ : 259-261; Jacques T. GODBOUT, dir., La participation politique. Leçons des dernières décennies, 46, 2 : 313-315 
PERIN, Roberto, Philippe PRÉVOST, $L a$ France et les nominations épiscopales au Canada de 1921 à 1940: un combat pour la francophonie, 50, 2: 300-302

PERRAULT, Stéphane, Alan C. CAIRNS, Charter Versus Federalism: the Dilemmas of Constitutional Reform, 46, 4: 672-674

PERREAULT, Daniel, Philip SMITH, Harvest from the Rock: a History of Mining in Ontario (NB), 41, 1: 108109

PERREAULT, Stéphane-D., Guy LAPOINTE, dir., Société, culture et religion à Montréal: $X I X^{e}-X X^{e}$ siècles, 48 , 4: 571-572

PERRON, Normand, Claude BEAUCHAMP, Agropur. Cinquante ans de rêves et de réalisations depuis la Société coopérative agricole du canton de Granby, 1938-1988, 42, 4: 598-600

PICHÉ, Claude, Jacques MATHIEU, dir., Les Plaines d'Abraham. Le culte de l'idéal, 47, 4: 570-571; Stanley G. TRIGGS, Le studio de William Notman. Objectif Canada/William Notman's Studio. The Canadian Picture, 47, 4: 578-579

PIETTE, Christine, Yolande COHEN, dir., Femmes et contre-pouvoirs, 42, 1 : 94-96

PIRES, Alvaro P., Jean-Marie FECTEAU, Un nouvel ordre des choses: la pauvreté, le crime, l'État au Québec, de la fin du XVIII siècle à 1840, 45, 1: 113-117

PLAMONDON, Lilianne, COLLECTIF INDISCIPLINE HISTORIQUE, Femmes et histoires: derrière les mots (NB), 41, 4: 638; Deborah COWLEY et George COWLEY, Portrait de Pauline Vanier, 49, 2: 263-265

POIRIER, Lucien, Gilles LEFEBVRE, $L a$ musique d'une vie (NB), 48, 4: 592-593

POIRIER, Marie, Michael BROWN, Jew or Juif? Jews, French Canadians, and Anglo Canadians, 1759-1914, 41, 2: 257-258; Micheline LABELLE, Geneviève TURCOTTE, Marianne KEM-
PENEERS et Deirdre MEINTEL, Histoire d'immigrées - Itinéraires d'ouvrières colombiennes, grecques, haïtiennes et portugaises de Montréal, 42, 1: 105107; John E. ZUCCHI, Italians in Toronto - Development of a National Identity, 1875-1935, 43, 4: 599-600

PORTER, John R., Mary Macaulay ALLODI et Rosemarie L. TOVELL, $A n$ Engraver's Pilgrimage. James Smillie in Quebec, 1821-1830, 43, 3: 403-405

PORTES, Jacques, Michel GRENON, dir., L'image de la Révolution française au Québec, 1789-1989, 44, 1: 111-112; Phillippe PRÉVOST, La France et le Canada, d'une après-guerre à l'autre (1918-1944), 48, 4: 581-582

POSTOLEC, Geneviève, édition critique par Pauline DUBÉ, Les frères insoumis ou "l'ombre d'un clocher» (NB), 50, 2: 320-321; Yves LANDRY, Orphelines en France, pionnières au Canada. Les Filles du roi au XVII siècle, 47, 3: 443444

PRÉVOS, André, Patrick GRIOLET, Cadjins et Créoles en Louisiane. Histoire et survivance d'une francophonie, 41, 1 : 77-79

PRÉVOS, André J. M., Carl A. BRASSEAUX, The Founding of New Acadia. The Beginnings of Acadian Life in Louisiana, 1765-1803, 41, 3: 422-423; Ronald CREAGH, Nos cousins d'Amérique. Histoire des Français aux ÉtatsUnis, 42, 4: 615-616; R. M. GOLDEN, dir., The Huguenot Connection: the Edict of Nantes, its Revocation, and Early French Migration to South $\mathrm{Ca}$ rolina, 42, 1: 98-100

PRITCHARD, James S., Bruce G. TRIGGER, Natives and Newcomers: Canada's "Heroic Age» Reconsidered, "L'Amérindien victime de l'incompétence des historiens» (NC), 41, 1: 6370; Denys DELÂGE, Le pays renversé. Amérindiens et Européens en Amérique $d u$ nord-est, 1600-1664, 41, 3: 409-411

PRONOVOST, Gilles, Gilles JANSON, Emparons-nous du sport. Les Cana- 
diens français et le sport au XIX siècle, 49, 4: 581-582; Yvan LAMONDE et Raymond MONTPETIT, Le parc Sohmer de Montréal 1889-1919. Un lieu populaire de culture urbaine, 41, 1: 9395

$\mathbf{R}$

RABOY, Marc, L'État et la culture (NB), 41, 1: 110-111

RAJOTTE-LaBREQUE, Marie-Paule, Majorique MARCHAND; documents présentés et annotés par Jean ROY et Christine HUDON, Le journal de Majorique Marchand, curé de Drummondville, 1865-1889, 48, 4: 575-577

RAMIREZ, Bruno, Barbara ROBERTS, Whence They Came: Deportation From Canada, 1900-1935, 43, 2: 273-275

REGNIER, Serge, Japp LINTVELT, Réal OUELLET et Hub HERMANS, dir., Culture et colonisation en Amérique du Nord: Canada, États-Unis, Mexique / Culture and Colonization in North America: Canada, United States, Mexico, 48, 4: 574-575

RHÉAUME, Gilles, Gaston CHOLETTE, L'Office de la langue française de 1961 à 1974, 48, 2: 247-249

RIOUX, Denise, Michael BLISS, Montréal au temps du grand fléau: l'histoire de l'épidémie de 1885, 48, 4: 543-544

ROBERT, Jean-Claude, Thérèse BEAUDOIN, L'été dans la culture québécoise $X V I I^{e}-X I X^{e}$ siècle, 41, 3: 417-419; PaulLouis MARTIN, La chasse au Québec, 45, 3: 453-455

ROBERT, Lucie, Marie-Andrée BEAU$\mathrm{DET}$, Langue et littérature au Québec, 1895-1914. L'impact de la situation linguistique sur la formation du champ littéraire. Essai, 46, 2: 289-291
ROBICHAUD, Léon, R. Louis GENTILCORE, dir., Atlas historique du Canada, 2: La transformation du territoire 1800-1891, 48, 4: 557-559; Daniel HICKEY et al., Guide des sources archivistiques sur l'industrie forestière $d u$ Nouveau-Brunswick (NB), 45, 3: 471472; Daniel HICKEY, dir., Moncton, 1871-1929. Changements socio-économiques dans une ville ferroviaire, 45,3 : 442-445

ROBIDOUX, Réjean, Maurice LEMIRE, dir., Le romantisme au Canada, 48, 2: 265-267

ROBILLARD, Denise, Philippe SYLVAIN et Nive VOISINE, Histoire $d u$ catholicisme québécois, II: Réveil et consolidation, 2: 1840-1898, 47, 3: 453-456

ROBY, Yves, Francophonie nord-américaine: bibliographie sélective (NB), 46, 2: 368-369; François WEIL, Les Franco-Américains, 1860-1980, 44, 2: 286288

ROCHER, Guy, Georges-Henri LÉVESQUE, Souvenances. Entretiens avec Simon Jutras, 3: Escales et parcours, 43, 4: 588-589; Arthur TREMBLAY; Robert BLAIS et Marc SIMARD, coll., Le ministère de l'Éducation et le Conseil supérieur. Antécédents et création, 1867-1964, 43, 3: 434-435

ROUILLARD, Jacques, Jean-Pierre COLLIN, La Ligue ouvrière catholique canadienne 1938-1954, 50, 2: 255-257; D. R. HOPKIN et G. S. KEALEY, Class, Community and the Labour Movement: Wales and Canada, 18501930, 43, 4: 576-578; Gregory S. KEALEY et Greg PATMORE, Canadian and Australian Labour History. Towards a Comparative Perspective, 45, 1: 119-121; Esdras MINVILLE, Syndicalisme, législation ouvrière et régime social au Québec avant 1940, 41, 4: 624-625; Max SWERDLOW, Brother Max: Labour Organizer and Educator, 45, 1: 119-121 
ROUSSEAU, François, Denis GOULET, François HUDON et Othmar KEEL, Histoire de l'Hôpital Notre-Dame de Montréal, 1880-1980, 48, 4: 563-565; Jules LAMBERT, Mille fenêtres, 50, 2: 283-284

ROUSSEAU, Louis, Lucien LEMIEUX, Histoire du catholicisme québécois. Les $X V I I I^{e}$ et XIX siècles, 1: Les années difficiles (1760-1839), 44, 4: 603-605

ROUSSEAU, Yvan, Ronald RUDIN, In Whose Interest? Quebec's Caisses populaires, 1900-1945, 44, 3: 450-452

ROY, Fernande, Jean De BONVILLE, $L a$ presse québécoise de 1884 à 1914. Genèse d'un média de masse, 43, 3: 407410; Marie-France FORTIER, Index du Bulletin des recherches historiques (NB), 43, 1: 128; Yvan LAMONDE, Gens de parole. Conférences publiques, essais et débats à l'Institut canadien de Montréal, 1845-1871, 45, 3: 448-450; UNION DES ÉCRIVAINS QUÉBÉCOIS; textes recueillis et présentés par Jean-Paul BERNARD, Assemblées publiques, résolutions et déclarations de 1837-1838., 43, 2: 282

ROY, Jean, Anne-Marie DESDOUITS, La vie traditionnelle au pays de Caux et au Canada français. Le cycle des saisons, 41, 4: 610-613; Louis ROUSSEAU, dir., Le bas clergé catholique au dix-neuvième siècle. Approche comparative d'une population pastorale en voie de changement, 50, 2: 304-306

RUDIN, Ronald, Yvan ROUSSEAU et Roger LEVASSEUR, Du comptoir au réseau financier: l'expérience historique du Mouvement Desjardins dans la région du centre du Québec, 1909 1970, 49, 3: 443-444; réplique, 50, 1: 132-135; réponse de Ronald RUDIN, 50, 1: 135-137; Fernande ROY, Histoire des idéologies au Québec aux $X I X^{e}$ et $X X^{e}$ siècles, 48, 2: 275-277; Margaret W. WESTLEY, Grandeur et déclin: l'élite anglo-protestante de Montréal, 1900-1950, 44, 4: 616-618
RUEL, Christian, Dale STANDEN et David McNAB, Gin Das Wingan: Documenting Aboriginal History in Ontario (NB), 50, 2: 324-325
SAINT-ONGE, Nicole, Thomas FLANAGAN, Metis Lands in Manitoba, 46, 3: 521-523

SAINT-PIERRE, Diane, Gaston DESCHÊNES, La Côte-du-Sud, cette inconnue, 46, 3: 517-519

SAINT-PIERRE, Jacques, Michel F. GIRARD, L'écologisme retrouvé: essor et déclin de la Commission de la conservation du Canada, 48, 4: 561-563

SAINT-PIERRE, Jocelyn, Jean De BONVILLE, Les quotidiens montréalais de 1945 à 1985: morphologie et contenu, 50, 3: 445-448

SANFAÇON, André, Jean-Claude DUBÉ et Pierre JULIEN-LAFERRIÈRE, Les Bigot du XVI siècle à la Révolution: évolution d'un lignage, 42, 2: 272-276; John H. ELLIOTT, Richelieu et Olivares, 46, 2: 307-309

SARRA-BOURNET, Michel, Robert COMEAU et Bernard DIONNE, Le droit de se taire. Histoire des communistes au Québec, de la Première Guerre mondiale à la Révolution tranquille, 44, 2: 263-265; Ramsay COOK, Canada, Quebec, and the Uses of $\mathrm{Na}$ tionalism (NB), 41, 1: 107-108; Yves LAVERTU, L'affaire Bernonville. Le Québec face à Pétain et à la Collaboration (1948-1951), 50, 2: 290-292; Claude-V. MARSOLAIS, Le référendum confisqué, 47, 2: 292-293

SAVARD, Marie-Élaine, Réjean ROBIDOUX, Fonder une littérature nationale: notes d'histoire littéraire, 48, 4: 582-584 
SAVARD, Pierre, Jean HAMELIN, dir., Les Franciscains au Canada, 18901990, 45, 1: 117-119; Sylvain SIMARD, Mythe et reflet de la France. L'image du Canada en France, 18501914, 42, 2: 304-307

SAVOIE, Sylvie, Françoise DEROYPINEAU, Jeanne Mance. De Langres à Montréal, la passion de soigner (NB), 50, 3: 484; Roger MAGNUSON, Education in New France, 47, 1: 128-130; Louis PELLETIER, Le clergé en Nouvelle-France. Étude démographique et répertoire bibliographique, 48, 4: 577579

SEAGER, Allen, Robert BOTHWELL, Ian DRUMMOND et John ENGLISH, Canada 1900-1945, 42, 2: 266-268

SENESE, Phyllis M., Giselle HUOT et al., Lionel Groulx: correspondance 1894-1967, 1: Le prêtre-éducateur, 44, 2: 271-273; Douglas V. VERNEY, Three Civilizations, Two Cultures, One State: Canada's Political Traditions, 41, 3: 428-432

SÉVIGNY, André, Jack VERNEY, The Good Regiment: the Carignan-Salières Regiment in Canada, 1665-1668, 45, 3: 468-470

SIMARD, Jean-Jacques, Marcel RIOUX, Un peuple dans le siècle, 44, 3: 444-446; Pierre TURGEON, La Radissonie. Le pays de la Baie James, 46, 2: 356-358

SIMARD, Sylvain, Gérard BERGERON, Quand Tocqueville et Siegfried nous observaient..., 45, 2: 261-263

SIOUI, Georges E., Daniel K. RICHTER et James H. MERREL, dir., Beyond the Covenant Chain: the Iroquois and their Neighbors in Indian North America, 1600-1800, 42, 1: 115-118

SMITH, Allan, G. E. D. MARTIN, Britain and the Origins of Canadian Confederation, 49, 3: 439-440; A. B. McKILLOP et Paul ROMNEY, dir., God's Peculiar Peoples: Essays on Political Culture in Nineteenth Century Canada, S. F. Wise , 48, 1: 109-111
SMITH, Donald B., Sarah CARTER, Lost Harvests, Prairie Indian Reserve Farmers and Government Policy, 45, 2: 268-270; Marc JETTEN, Enclaves amérindiennes: les «réductions» du Canada, 1637-1701, 49, 3: 436-438

SMITH, Miriam, Christian DUFOUR, La rupture tranquille, 47, 1: 114-117

ST-GEORGES, Lise, Émilia CHICOINE, La métairie de Marguerite Bourgeoys à la Pointe-Saint-Charles (NB), 42, 1 : 125; Devenir chercheur-e: itinéraires et perspectives, 41, 4: 631-632

ST-HILAIRE, Marc, Bruno RAMIREZ, Par monts et par vaux. Migrants canadiens-français et italiens dans l'économie nord-atlantique, 1860-1914, 47, 4: 574-577

STANDEN, S. Dale, Luc-Normand TELLIER, Face aux Colbert: les Le Tellier, Vauban, Turgot... et l'avènement du libéralisme, 43, 3: 431-433

STAPINSKY, Stéphane, Yvan LAMONDE, La librairie et l'édition à Montréal 1776-1920, 45, 3: 451-453

STRONG-BOAG, Veronica, Suzanne MORTON, Ideal Surroundings. Domestic Life in a Working-Class Suburb in the 1920's, 49, 4: 589-591

SUTTO, Claude, Henry HELLER, Iron and Blood. Civil Wars in SixteenthCentury France, 45, 3: 440-442

SWEENY, Robert, Peter BASKERVILLE, dir., The Bank of Upper Canada. A Collection of Documents, 43, 1 : 102-104

SWEENY, Robert C. H., Philip A. BUCKNER et John G. REID, dir., The Atlantic Region to Confederation: a History, 48, 4: 548-550; Graham D. TAYLOR et Peter A. BASKERVILLE, A Concise History of Business in $\mathrm{Ca}$ nada, 50, 2: 307-309 
$\mathbf{T}$

TAILLEMITE, Étienne, Nicole CHAMBERLAND, Jane McLEOD, Christine TURGEON et Raymonde LITALIEN, dir., Amirauté de Guyenne, source de l'histoire de la Nouvelle-France: (série 6B), Archives départementales de la Gironde, Bordeaux, France (NB), 48, 1: 119

TASCHEREAU, Sylvie, Denise HELLY, Les Chinois à Montréal, 1877-1951, 42, 1: 102-105; L'histoire de l'immigration au Québec: une invitation à fuir les ghettos (NC), 41, 4: 575-589

TÉTREAULT, Martin, Denise RIOUX, La grippe espagnole à Sherbrooke et dans les Cantons-de-l'Est, 48, 1: 116-118

THÉRIO, Adrien, Éliane GUBIN et Yvan LAMONDE, Un Canadien français en Belgique au XIX siècle? Correspondance d'exil de L.-A. Dessaulles, 18751878, 47, 1: 117-118

THOMAS, Gerald, Gary R. BUTLER, Saying Isn't Believing. Conversation, Narrative and the Discourse of Belief in a French Newfoundland Community, 46, 2: 296-298

THOMPSON, John Herd, Alan METCALFE, Canada Learns to Play: the Emergence of Organized Sport in Canada, 1807-1914, 43, 3: 419-421; J. R. MILLER, Skyscrapers Hide the Heavens: a History of Indian-White Relations in Canada, 44, 2: 276-279

TOUSIGNANT, Claude, Noël CORBETT, dir., Langue et identité. Le français et les francophones d'Amérique du Nord, 45, 4: 606-609

TOUSIGNANT, Pierre, David J. BERCUSON, Kerry M. ABEL, Donald H. AKENSON, Peter A. BASKERVILLE, John M. BUMSTED et John G. REID, COLONIES: Canada to 1867, 47, 1: 93-96; John HARE, Aux origines $d u$ parlementarisme québécois, 1791 -
1793. Étude et documents, 48, 3: 441443: Philip LAWSON, The Imperial Challenge. Quebec and Britain in the Age of the American Revolution, 44, 3: 435-438; Philip A. BUCKNER, The Transition to Responsible Government: British Policy in British North America, 181.5-1850, "La problématique du gouvernement responsable vue dans une nouvelle perspective historique» (NC), 42, 2: 253-261; Jack VERNEY, O'Callaghan: the Making and Unmaking of a Rebel, 50, 3: 478-481

TREMBLAY, Robert, Bernard DIONNE, Le syndicalisme au Québec, 47, 1: 112114

TREMBLAY, Rodrigue, Irwin GILLESPIE, Tax, Borrow and Spend. Financing Federal Spending in Canada, 1867-1990, 46, 3: 530-533

TRÉPANIER, Pierre, Louis BALTHAZAR, Bilan du nationalisme au Québec, 41, 3: 426-428; David J. BERCUSON et J. L. GRANATSTEIN, The Coliins Dictionary of Canadian History. 1867 to the Present (NB), 42, 4: 640; Manon BRUNET, Yolande BUONO, Yvan LAMONDE et André VANASSE, Bibliographie des études québécoises sur l'imprimé, 1970-1987 (NB), 45, 1: 128; COLLECTIF, McGill. A Celebration (NB), 45, 2: 297; Yves CORMIER, Les Aboiteaux en Acadie hier et aujourd'hui, 44, 2: 267-268; Henriette DESSAULLES, Journal, édition critique, 44, 1: 107108; Raoul DIONNE, La colonisation acadienne au Nouveau-Brunswick, 1760-1860. Données sur les concessions de terres, 44, 2: 267-268; C. Stewart DOTY, Acadian Hard Times. The Farm Security Administration in Maine's St.John Valley, 1940-1943 (NB), 45, 1: 129; Francis R. DOUGLAS, Richard JONES et Donald B. SMITH, Destinies. Canadian History Since Confederation, 42, 4: 626-629; Francis R. DOUGLAS, Richard JONES et Donald B. SMITH, Origins. 
Canadian History to Confederation, 42, 4: 626-629; L'Église et la question sociale de Léon XIII à Jean-Paul II (NB), 45, 4: 634-635; J. L. FINLAY et D. N. SPRAGUE, The Structure of $\mathrm{Ca}$ nadian History, 43, 4: 570-572; Raymond HUDON et Réjean PELLETIER, dir., L'engagement intellectuel. Mélanges en l'honneur de Léon Dion, 47, 2: 280-281; Yvan LAMONDE, Territoires de la culture québécoise (NB), 45 , 1: 130; Robert MAJOR, Jean Rivard ou l'art de réussir. Idéologies et utopie dans l'œuvre d'Antoine Gérin-Lajoie, 45, 4: 611-612; Johnny MONTBARBUT, Si l'Amérique française m'était contée. Essor et chute. Récit historique, 45, 1: 131; Pascal POIRIER, Causerie memramcookienne, 44, 2: 267-268; Jacques PORTES, dir., Le fait français et l'histoire du Canada, XIX $-X X^{e}$ siècle, 45, 3: 458-459; Martin ROBIN, Shades of Right. Nativist and Fascist Politics in Canada, 1920-1940, 46, 2: 348-350; Franklin TOKER, The Church of Notre-Dame in Montreal. An Architectural History (NB), 45, 2: $297-$ 298

TRUDEL, Marcel, Raymonde LITALIEN, Les explorateurs de l'Amérique $d u$ Nord, 1492-1795, 47, 4: 568-569

TRUDEL, Pierre, F. Richard SALISBURY, A Homeland for the Cree. Regional Development in James Bay 19711981, 41, 3: 447-449

TURGEON, Laurier, James S. PRITCHARD, Louis XV's Navy 1748-1762: a Study of Organization and Administration, 43, 4: 593-597

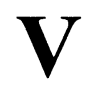

VALLIÈRES, Marc, Norman R. BALL, dir., Bâtir un pays. Histoire des travaux publics au Canada, 42, 4: 597-598; Robert Malcolm CAMPBELL, Grand Illusions: the Politics of the Keynesian Experience in Canada, 1945-1975, 42, 1: 89-91; James Iain GOW, Histoire de l'administration publique québécoise, 1867-1970, 40, 3: 432-434; René HARDY, La sidérurgie dans le monde rural: les hauts fourneaux du Québec au XIX siècle, 50, 3: 456-458; John HARE, Marc LAFRANCE et DavidThiery RUDDEL, Histoire de la ville de Québec, 1608-1871, 42, 1: 100-102; G. KURGAN-VAN HENTENRYK et J. LAUREYSSENS, Un siècle d'investissements belges au Canada, 41, 2: 271-273; T. D. REGEHR, The Beauharnois Scandal: a Story of Canadian Entrepreneurship and Politics, 44, 1: 117-119

VERDON, Michel, Georges AUGUSTINS, Comment se perpétuer? Devenir des lignées et destins des patrimoines dans les paysanneries européennes, 45 , 2: 257-259

VERMEIRRE, André, Ginette KURGANVAN HENTENRYK, dir., La question sociale en Belgique et au Canada XIX ${ }^{e}$ XXe siècles, 43, 3: 411-412

VÉRONNEAU, Pierre, Jocelyne DENAULT, Dans l'ombre des projecteurs: les Québécoises et le cinéma, 50, 2: 262-263; Yves LEVER, Histoire générale du cinéma au Québec, 42, 4: 632634

VERRETTE, Michel, Pierre LANTHIER et Guildo ROUSSEAU, dir., La culture inventée. Les stratégies culturelles aux 19 e et 20e siècles, 46, 3: 537-538;

VIGOD, Bernard L., Le Québec contemporain vu par Paul-André Linteau, René Durocher, Jean-Claude Robert et François Ricard (NC), 41, 2: 237-243

VINCENT, Odette, COLLECTIF, Thetford Mines à ciel ouvert. Histoire d'une ville minière 1892-1992, 50, 1: 107-109

VINCENT-DOMEY, Odette, David M. RAYSIDE, A Small Town in Modern 
Times: Alexandria, Ontario, 45, 3: 462464

VOISINE, Nive, Yvan LAMONDE, Louis-Antoine Dessaulles (1818-1895). Un seigneur libéral et anticlérical, 48, 4: 570-571; René LATOURELLE, Jean de Brébeuf, 48, 1: 100-102; Rolland LITALIEN, dir., Le Grand séminaire de Montréal de 1840 à 1990: 150 ans au service de la formation des prêtres, 44, 4: 606-607; Roberto PERIN, Rome in Canada. The Vatican and $\mathrm{Ca}$ nadian Affairs in the Late Victorian Age, 44, 2: 281-283; Religion/Culture. Comparative Canadian Studies - Études canadiennes comparées. Canadian Issues/Thèmes canadiens (NB), 41, 4: 635-636

W

WATELET, Hubert, Donald FISHER, Les sciences sociales au Canada. 50 ans d'activités à l'échelle nationale par la Fédération canadienne des sciences sociales, 47, 2: 271-273; François LEBRUN et Normand SÉGUIN, Sociétés villageoises et rapports villes-campagnes au Québec et dans la France de l'Ouest, $X V I T-X X^{e}$ siècles, "Ombres et lumières d'un vaste projet: l'histoire rurale comparée de la France de l'Ouest et du Québec» (NC), 43, 2: 233-245

WHITAKER, Reg, Larry HANNANT, The Infernal Machine: Investigating the Loyalty of Canada's Citizens, 50, 2: 278-279

WHITE, Bruce, Peter S. SCHMALZ, The Ojibwa of Southern Ontario, 46, 2: 350-352

WIEN, Thomas, Gérard BOUCHARD, Quelques arpents d'Amérique. Population, économie, famille au Saguenay 1838-1971 (DEB), 50, 3: 401-435; Bar- ry M. GOUGH, The Journal of Alexander Henry the Younger 17991814, 1: Red River and the Journey to the Missouri, 43, 4: 573-574; Jacques MATHIEU et Alain LABERGE, dir., L'occupation des terres dans la vallée du Saint-Laurent. Les aveux et dénombrements, 1723-1745 (NB), 46, 2: 374; Dale MIQUELON, New France 17011744. "A Supplement to Europe», 42, 3: 474-476; Rosemary E. OMMER, dir., Merchant Credit and Labour Strategies in Historical Perspective, 45, 4: 614-617; Kathryn A. YOUNG, Kin, Commerce, Community. Merchants in the Port of Quebec, 1717-1745 (NB), 50, 3: 486-487

YOUNG, Brian, Dave McINTOSH, Les receveurs: histoire des Douanes et de l'Accise au Canada, 41, 2: 268-269; Jim PHILLIPS, Tina LOO et Susan LEWTHWAITE, Essays in the History of Canadian Law, 5: Crime and Criminal Justice, 50, 3: 472-474

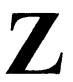

ZUCCHI, John E., Roberto PERIN et Franc STURINO, Arrangiarsi: the Italian Immigration Experience in Canada $, 43,4: 591-593$ 